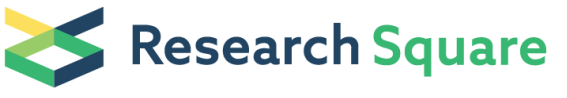 \\ Preprints are preliminary reports that have not undergone peer review. \\ They should not be considered conclusive, used to inform clinical practice, \\ or referenced by the media as validated information.
}

\section{Comparison of Different Anthropometric Indices to Predict Prediabetes in North China, Beijing: a 7-year Prospective Study}

\section{Yulin Gu}

Chinese PLA General Hospital

Changming Zhao

Chinese PLA General Hospital

\section{Ping An}

Chinese PLA General Hospital

\section{Kang Chen}

Chinese PLA General Hospital

\section{Yijun Li}

Chinese PLA General Hospital

\section{Li Zang}

Chinese PLA General Hospital

\section{Weijun Gu}

Chinese PLA General Hospital

\section{Jingtao Dou}

Chinese PLA General Hospital

Zhaohui Lyu

Chinese PLA General Hospital

\section{Jianming $\mathrm{Ba}$}

Chinese PLA General Hospital

Yiming Mu ( $\square$ muyiming@301hospital.com.cn )

Chinese PLA General Hospital https://orcid.org/0000-0002-3344-3540

\section{Original investigation}

Keywords: Prediabetes, Body adiposity index, Overall obesity, Central obesity

Posted Date: June 22nd, 2020

DOI: https://doi.org/10.21203/rs.3.rs-36839/v1

License: (c) (i) This work is licensed under a Creative Commons Attribution 4.0 International License. Read Full License 


\section{Abstract \\ Background}

Obesity is an increasing problem worldwide and is one of the underlying risk factors for prediabetes. Although WHtR, BMI, WC and WHR were found to be associated with dysglycemia, in view of significant differences in basic characteristics, glycemic metabolism, and ethnicity, it is of practical value to investigate which indicators are the most useful measures to predict the incidence of prediabetes in Chinese population. The aim of this study was to compare the value of different anthropometric measures of obesity in the detection of prediabetes in a cohort study in China and to identify the best cut-off point of predicting prediabetes in this population.

\section{Methods}

The present study was a part of the ongoing Risk Evaluation of cAncers in Chinese diabetic Individuals: an IONgitudinal (REACTION) study. After 7 years of follow-up, a total of 2568 individuals in Beijing were analyzed. Waist-to-height ratio (WHtR), body mass index (BMI), waist circumference (WC) and waist-to-hip ratio (WHR) were measured at baseline and during follow-up. These four values were divided into tertiles. Fast blood glucose and postprandial blood glucose were tested. The association of these four anthropometric values with blood glucose were tested using multiple logistic analysis.

\section{Results}

A total of 2568 normoglycemic participants (810 males and 1758 females) were recruited, with an average age of $62.68 \pm 6.97$ years. After 7 years of follow-up, 52 subjects had developed newly diagnosed diabetes, and 423 individuals had developed prediabetes. Among those with prediabetes, 278 had IGT, 105 had IFG, and 40 had IGT + IFG. The incidences of prediabetes and diabetes were calculated to be 115.3 per 1000 person-years and 14.2 per 1000 person-years, respectively. Multivariable regression analysis revealed that there was no significant difference in the WHtR, BMI, WC, WHR among those with diabetes and nondiabets. In the general population and the group of females with prediabetes, we found that WHtR was the best value to predict prediabetes, and with an increase in the WHtR, the possibility of prediabetes increased by $90 \%$ or $102 \%$, respectively. In the group of males, WHR was the most associated with prediabetes, and people in Tertile 2 was 2.62 times more likely to suffer from prediabetes than those in Tertile 1. For females, the optimal cut-off points of WHtR, BMI, WC and WHR were $0.48,23.5 \mathrm{~kg} / \mathrm{m}^{2}, 75.6 \mathrm{~cm}$ and 0.84 , respectively. For males, the optimal cut-off points of the WHtR, BMI, WC and WHR were $0.50,25.5 \mathrm{~kg} / \mathrm{m}^{2}, 88.9 \mathrm{~cm}$ and 0.93 , respectively.

\section{Conclusion}

In a Chinese study population, WHtR was the best predictor of the development of prediabetes in the general population and in females 7 years in advance, while WHR could predict the development of prediabetes in males. Early identification of prediabetes can better prevent diabetes.

\section{Background}

The prevalence of prediabetes is rapidly increasing worldwide. Among Chinese adults, the overall prevalence of

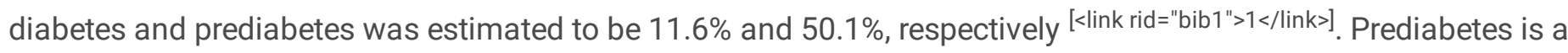


warning sign of diabetes. Approximately $75 \%-80 \%$ of patients with diabetes ultimately develop cardiovascular disease, and patients with prediabetes have also been shown to have an increased risk of heart attack and stroke $e^{[2-4]}$. Measures to prevent prediabetes are critical.

Obesity is an increasing problem worldwide and is one of the underlying risk factors for metabolic diseases, such as dysglycemia, dyslipidemia, hypertension, and cardiovascular disease. Obesity can be identified by many indicators to identify obesity, such as the body mass index (BMI), waist-to-height ratio (WHtR), waist-to-hip ratio (WHR) and waist circumference (WC). BMI was the most commonly used indictor for defining obesity, which is recommended by World Health Organization (WHO). While studies conducted subsequently found that BMI can neither distinguish between muscle and fat body mass ${ }^{[5]}$ nor reflect abdominal fat. Some studies also have shown that increased BMI was not associated with increased mortality ${ }^{[6-10]}$. The studies available to date still have no conclusion as to which anthropometric parameter best predicts mortality. WC or WHR is encouraged for the additional use of BMI ${ }^{[11]}$. Recently, new anthropometric indices such as WHtR have been proposed as alternative indicators of obesity and can predict health risks and substantial health risks.

Although WHtR, BMI, WC and WHR were found to be associated with dysglycemia, in view of significant differences in basic characteristics, glycemic metabolism, and ethnicity ${ }^{[12-15]}$, it is of practical value to investigate which indicators are the most useful measures to predict the incidence of prediabetes in Chinese population. In this study, we compared the value of different anthropometric measures of obesity in the detection of prediabetes in a cohort study in China, aiming to explore the ability of these anthropometric indices to predict prediabetes and to identify the best cut-off point in this population.

\section{Methods}

\section{Study participants}

The present work was a part of the survey from the REACTION study. All subjects participated in a community longitudinal survey, which was conducted in the communities of Shijingshan district, Beijing, China. A total of 19443 residents were recruited and invited to participate in a questionnaire survey in 2011. The inclusion criteria were as follows: a, patients with normal blood glucose levels; and b, patients without diabetes, stroke, chronic heart disease or hyperlipidemia. The exclusion criteria were as follows: a, the presence of primary diseases at baseline, such as diabetes, heart infarction, stroke or chronic heart disease; $b$, the use of lipid-lowering medication or sleeping pills; $c$, the unavailability of demographic data at baseline; and d, outlier data. Follow-up visits for the subjects were conducted in 2015 and 2018, respectively. Finally, a total of 2568 participants were enrolled in the present study.

\section{Data Collection}

A questionnaire, designed by professional, was used to collect basic information, including age, sex, education, occupation, lifestyle, exercise, sleep time, marital status, education, a history of cardiovascular diseases, tumor, diabetes, hypertension, hyperlipidemia, acute/chronic nephritis, alcohol intake, smoking habits and tea habits. All investigators were trained with standard method about asking questionnaire and measuring before carrying out the investigation.

Blood samples were collected in the morning after at least $8 \mathrm{~h}$ of fasting overnight. $75 \mathrm{~g}$ oral glucose tolerance test or $100 \mathrm{~g}$ steamed-bread meal test was conducted based on different blood glucose levels. Blood glucose [including fasting blood glucose (FBG) and $2 \mathrm{~h}$ postload blood glucose (PBG)], blood lipids [including total cholesterol (TC), serum triglycerides (TG), low-density lipoprotein cholesterol (LDL-C) and high-density lipoprotein cholesterol (HDL-C)], gamma- 
glutamyl transferase (GGT), Cr and hemoglobin A1c (HbA1c) were measured. Blood samples were measured using an autoanalyzer (Cobas 8000 modular analyzer series: Roche Diagnostics, Basel, Switzerland). HbA1c was determined by HPLC using the VARIANT II Hemoglobin Testing System (Tosoh, Tokyo, Japan).

Blood pressure (BP), heart rate, height, weight, waist circumference and hip circumference were measured and recorded. BP and heart rate was measured three times by the same observer after the five-minute-resting. The average of the three figures was used for the analysis. All participants wear light clothing and without shoes to conduct anthropometric measurements after an 8 to $10 \mathrm{~h}$ overnight fast on the morning. The results were recorded to the nearest $0.1 \mathrm{~kg}$ or $0.1 \mathrm{~cm}$. Height was measured using the standard steel strip stadiometers. Weight was measured using the calibrated weighing scales. WC and hip circumference $(\mathrm{HC})$ were made using tape measures. WC was measured at the midpoint between the costal border and the iliac crest. Hip circumference was measured around the widest portion of the buttocks. BMI was calculated as the weight $(\mathrm{kg})$ divided by the height in meters squared $\left(\mathrm{m}^{2}\right)$. WHtR was calculated as the WC $(\mathrm{cm})$ divided by the height $(\mathrm{cm})$. WHR was calculated as the WC $(\mathrm{cm})$ divided by the $\mathrm{HC}(\mathrm{cm})$.

\section{Definitions}

Marital status was categorized into two levels: married and not married/widowed. Education was categorized into five levels: illiteracy, primary school, junior high school, senior high school and college. Current smoking: smoking occasionally or smoking more than once a week in the past 12 months. Current alcohol consumption: drinking occasionally or drinking more than once a week in the past 12 months. Current tea consumption: drinking tea occasionally or drinking tea more than once a week in the past 12 months. Regular exercise: engaging in moderate or vigorous exercise more than once a week. Blood glucose definitions were as follows: for diabetes, FBG $\geq 7.0 \mathrm{mmol} / \mathrm{L}$ or $P B G \geq 11.1 \mathrm{mmol} / \mathrm{L}$ or any self-reported history of diabetes; for prediabetes, 3 subgroups were defined as follows: impaired glucose tolerance (IGT): $\mathrm{FBG}<6.1 \mathrm{mmol} / \mathrm{L}$ and $7.8 \leq \mathrm{BPG}<11.1 \mathrm{mmol} / \mathrm{L}$, impaired fasting glucose (IFG): 6.1 $\leq \mathrm{FBG}<7.0 \mathrm{mmol} / \mathrm{L}$ and $\mathrm{BPG}<7.8 \mathrm{mmol} / \mathrm{L}$, and $\mathrm{IFG}+\mathrm{IGT}: 6.1 \leq \mathrm{FBG}<7.0 \mathrm{mmol} / \mathrm{L}$ and $7.8 \leq \mathrm{BPG}<11.1 \mathrm{mmol} / \mathrm{L}$; and for nondiabetes: $F B G<6.1$ and $P B G<7.8 \mathrm{mmol} / \mathrm{L}$ simultaneously.

\section{Statistical analysis}

Statistical analysis was performed using SPSS software (version 21.0.0.0). Continuous variables with normal distribution are presented as the mean \pm standard deviation (SD), while data skewed distribution are presented as the median (Q1, Q3). Categorical data are recorded as $\mathrm{n}$ and proportions. Student's t test, ANOVA and chi-squared tests were performed to test for significant differences in the general characteristics between the groups. Pearson correlation analysis was used to analyze the correlation coefficient. The odds ratio values (OR values) and the corresponding $95 \%$ confidence intervals (Cls) after adjusting confounding factors in different models were calculated using multivariate logistic regression. Finally, receiver operating characteristic (ROC) curve was used to analyze the discrimination ability and to determine the best cut-off value. Sensitivity and specificity were calculated based on cut-off values, which were estimated using the maximized Youden index.

\section{Results}

\section{Characteristics of participants at baseline}

Totally, 2568 normoglycemic participants (810 males and 1758 females) were recruited in 2011 (Fig. 1), with an average age of $62.68 \pm 6.97$ years. After 7 years of follow-up, 52 subjects had developed newly diagnosed diabetes, and 423 individuals had developed prediabetes. Among participants with prediabetes, 278 had IGT, 105 had IFG, and 40 had IGT + IFG. The incidences of prediabetes and diabetes were 115.3 per 1000 person-years and 14.2 per 1000 person- 
years, respectively. As can be seen in Table 1 and Table 2, the average age of subjects who developed diabetes (64.06 \pm 5.23 years old) or prediabetes $(64.06 \pm 6.94$ years old) was older than that of participants who did not develop diabetes ( $62.65 \pm 7.04$ years old and $62.36 \pm 6.98$ years old). As expected, the levels of systolic pressure, WHtR, weight, $\mathrm{BMI}, \mathrm{WC}, \mathrm{WHR}, \mathrm{TG}, \mathrm{FBG}, \mathrm{PBG}$ and $\mathrm{HbA} 1 \mathrm{c}$ among newly diagnosed diabetes patients were more likely to be higher than the levels among those who did not develop diabetes $(p=0.019, p=0.001, p=0.002, p=0.001, p=0.001, p=0.004, p=$ $0.002, p<0.001, p=0.014$ and $p<0.001)$. While the result of HDL-c was the opposite $(p<0.001)$. Moreover, the family history of diabetes was significantly different among different groups $(p=0.046)$. The rate of current employment was much lower while the rate of regular exercise and diabetes family history were much higher in the group of people who had developed prediabetes than in the other groups (Table 2). The levels of WHtR $(p<0.001)$, weight $(p<0.001)$, BMI ( $p$ $<0.001)$, WC $(p<0.001), H C(p=0.016)$, WHR $(p<0.001)$, systolic pressure $(p<0.001)$, diastolic pressure $(p=0.001), T G$ $(<=0.001), \operatorname{LDL}-\mathrm{c}(\mathrm{p}=0.008)$, FBG $(p<0.001)$, PBG $(p<0.001)$ and HbA1c $(p<0.001)$ among those with prediabetes were more likely to be higher than those among people who did not develop prediabetes. The result of HDL-c was also the opposite $(p=0.002)$. 
Table 1

Baseline characteristics of study population grouped by diabetes and nondiabetes

\begin{tabular}{|c|c|c|c|c|c|}
\hline & & Total $(n=2568)$ & Diabetes $(n=52)$ & $\begin{array}{l}\text { Nondiabets }(n= \\
\text { 2516) }\end{array}$ & $\begin{array}{l}p- \\
\text { value }\end{array}$ \\
\hline Age(years old) & & $62.68 \pm 6.97$ & $64.06 \pm 5.23$ & $62.65 \pm 7.04$ & $\begin{array}{l}p= \\
0.149\end{array}$ \\
\hline \multirow[t]{2}{*}{ Gender } & male & $810(31.54 \%)$ & $20(38.46 \%)$ & 790(31.40\%) & \multirow{2}{*}{$\begin{array}{l}p= \\
0.293\end{array}$} \\
\hline & female & $1758(68.46 \%)$ & $32(61.54 \%)$ & $1726(68.60 \%)$ & \\
\hline \multirow[t]{2}{*}{ Marital status(n, \%) } & married & 2433(94.74\%) & $48(92.31 \%)$ & 2385(94.79\%) & \multirow{2}{*}{$\begin{array}{l}p= \\
0.350\end{array}$} \\
\hline & $\begin{array}{l}\text { not married } \\
\text { / widowed }\end{array}$ & $135(5.26 \%)$ & $4(7.69 \%)$ & $131(5.21 \%)$ & \\
\hline \multirow{2}{*}{$\begin{array}{l}\text { Current employment(n, } \\
\%)\end{array}$} & yes & $591(23.01 \%)$ & $6(11.54 \%)$ & $585(23.25 \%)$ & \multirow{2}{*}{$\begin{array}{l}p= \\
0.065\end{array}$} \\
\hline & no & 1977(76.99\%) & $46(88.46 \%)$ & 1931(76.75\%) & \\
\hline \multirow[t]{5}{*}{ Education(n, \%) } & illiteracy & $17(0.66 \%)$ & $1(1.92 \%)$ & $16(0.64 \%)$ & \multirow{5}{*}{$\begin{array}{l}p= \\
0.209\end{array}$} \\
\hline & primary schlool & $72(2.80 \%)$ & $1(1.92 \%)$ & $72(2.86 \%)$ & \\
\hline & $\begin{array}{l}\text { junior high } \\
\text { school }\end{array}$ & 737(28.70\%) & $20(38.46 \%)$ & $716(28.46 \%)$ & \\
\hline & $\begin{array}{l}\text { senior high } \\
\text { school }\end{array}$ & $1237(48.17 \%)$ & $19(36.54 \%)$ & $1218(48.41 \%)$ & \\
\hline & college & $505(19.67 \%)$ & 11(21.16\%) & 494(19.63\%) & \\
\hline \multirow[t]{2}{*}{ Current smoker(n, \%) } & yes & $471(18.34 \%)$ & $12(23.08 \%)$ & $459(18.24 \%)$ & \multirow{2}{*}{$\begin{array}{l}p= \\
0.469\end{array}$} \\
\hline & no & 2097(81.66\%) & $40(76.92 \%)$ & 2057(81.76\%) & \\
\hline \multirow{2}{*}{$\begin{array}{l}\text { Current alcohol } \\
\text { consumption(n, \%) }\end{array}$} & yes & $803(31.27 \%)$ & $15(28.85 \%)$ & 788(31.32\%) & \multirow{2}{*}{$\begin{array}{l}p= \\
0.764\end{array}$} \\
\hline & no & 1765(68.73\%) & $37(71.15 \%)$ & 1728(68.68\%) & \\
\hline \multirow{2}{*}{$\begin{array}{l}\text { Current tea } \\
\text { consumption(n, \%) }\end{array}$} & yes & $1647(64.14 \%)$ & $38(73.08 \%)$ & $1609(63.95 \%)$ & \multirow{2}{*}{$\begin{array}{l}p= \\
0.191\end{array}$} \\
\hline & no & $921(35.86 \%)$ & $14(26.92 \%)$ & $907(36.05 \%)$ & \\
\hline \multirow[t]{2}{*}{ Regular exercise(n, \%) } & yes & $282(10.98 \%)$ & $5(9.62 \%)$ & $277(11.01 \%)$ & \multirow{2}{*}{$\begin{array}{l}p= \\
0.829\end{array}$} \\
\hline & no & $2286(89.02 \%)$ & $47(90.38 \%)$ & 2239(88.99\%) & \\
\hline sleeptime(h) & & $7.5(7.0,8.0)$ & $8.0(7.0,8.38)$ & $7.5(7.0,8.0)$ & $\begin{array}{l}p= \\
0.868\end{array}$ \\
\hline \multirow[t]{2}{*}{ Diabetes family history } & yes & $588(22.90 \%)$ & 18(34.62\%) & $570(22.66 \%)$ & \multirow{2}{*}{$\begin{array}{l}p= \\
0.046 *\end{array}$} \\
\hline & no & 1980(77.10\%) & $34(65.38 \%)$ & 1946(77.34\%) & \\
\hline
\end{tabular}

Data were mean $\pm S D$ or median (IQR) for skewed variables or numbers (proportions) for categorical variables

* indicate significance of $P$ value $(P<0.05)$

SBP: systolic blood pressure; DBP: diastolic blood pressure; GGT: gamma-glutamyl transferase; TC: total cholesterol; TG: triglycerides; HDL-C: high-density lipoprotein cholesterol; LDL-C: low-density lipoprotein cholesterol; FBG: fasting blood glucose; PBG: postprandial blood glucose; HbA1c: hemoglobin A1c. 


\begin{tabular}{|c|c|c|c|c|}
\hline & Total $(n=2568)$ & Diabetes $(n=52)$ & $\begin{array}{l}\text { Nondiabets }(n= \\
2516)\end{array}$ & $\begin{array}{l}p- \\
\text { value }\end{array}$ \\
\hline $\mathrm{SBP}(\mathrm{mmHg})$ & $\begin{array}{l}\text { 126.67(117.00, } \\
137.00)\end{array}$ & $\begin{array}{l}\text { 130.17(122.50, } \\
142.50)\end{array}$ & $\begin{array}{l}\text { 126.33(117.00, } \\
137.67)\end{array}$ & $\begin{array}{l}\mathrm{p}= \\
0.019 *\end{array}$ \\
\hline $\mathrm{DBP}(\mathrm{mmHg})$ & $\begin{array}{l}74.67(68.33, \\
80.75)\end{array}$ & $\begin{array}{l}\text { 78.00(70.08, } \\
82.17)\end{array}$ & $\begin{array}{l}\text { 74.67(68.33, } \\
80.67)\end{array}$ & $\begin{array}{l}\mathrm{p}= \\
0.075\end{array}$ \\
\hline Pulse(bp) & $\begin{array}{l}\text { 76.33(70.00, } \\
83.67)\end{array}$ & $\begin{array}{l}76.83(70.75 \\
84.00)\end{array}$ & $\begin{array}{l}\text { 76.33(70.00, } \\
83.67)\end{array}$ & $\begin{array}{l}p= \\
0.549\end{array}$ \\
\hline WHtR & $0.50(0.47,0.53)$ & $0.52(0.49,0.56)$ & $0.50(0.47,0.53)$ & $\begin{array}{l}\mathrm{p}= \\
0.001 *\end{array}$ \\
\hline Height(cm) & $\begin{array}{l}161,00(156.00 \\
167.00)\end{array}$ & $\begin{array}{l}\text { 160.50(157.00, } \\
167.30)\end{array}$ & $\begin{array}{l}\text { 161.00(156.00, } \\
167.00)\end{array}$ & $\begin{array}{l}p= \\
0.667\end{array}$ \\
\hline Weight(kg) & $\begin{array}{l}64.00(58.00 \\
72.08)\end{array}$ & $\begin{array}{l}70.00(60.75 \\
77.75)\end{array}$ & $\begin{array}{l}64.00(58.00 \\
72.00)\end{array}$ & $\begin{array}{l}p= \\
0.002 *\end{array}$ \\
\hline BMI & $\begin{array}{l}24.77(22.78 \\
27.08)\end{array}$ & $\begin{array}{l}26.31(24.20 \\
28.55)\end{array}$ & $\begin{array}{l}24.76(22.74 \\
27.04)\end{array}$ & $\begin{array}{l}\mathrm{p}= \\
0.001 *\end{array}$ \\
\hline WC(cm) & $\begin{array}{l}81.00(75.00 \\
86.00)\end{array}$ & $\begin{array}{l}85.00(79.25 \\
90.88)\end{array}$ & $\begin{array}{l}80.55(75.00 \\
86.00)\end{array}$ & $\begin{array}{l}\mathrm{p}= \\
0.001 *\end{array}$ \\
\hline $\mathrm{HC}(\mathrm{cm})$ & $\begin{array}{l}92.00(89.00 \\
97.00)\end{array}$ & $\begin{array}{l}92.00(90.00 \\
100.00)\end{array}$ & $\begin{array}{l}92.00(89.00 \\
96.50)\end{array}$ & $\begin{array}{l}p= \\
0.056\end{array}$ \\
\hline WHR & $0.87(0.83,0.91))$ & $0.89 \pm 0.06$ & $0.87(0.83,0.91)$ & $\begin{array}{l}\mathrm{p}= \\
0.004^{*}\end{array}$ \\
\hline GGT & $\begin{array}{l}17.75(13.50 \\
25.00)\end{array}$ & $\begin{array}{l}22.80(18.20 \\
37.65)\end{array}$ & $\begin{array}{l}\text { 17.60(13.50, } \\
24.80)\end{array}$ & $\begin{array}{l}\mathrm{p}< \\
0.001 *\end{array}$ \\
\hline $\mathrm{Cr}$ & $\begin{array}{l}63.70(56.40 \\
74.00)\end{array}$ & $\begin{array}{l}62.00(56.20 \\
74.65)\end{array}$ & $\begin{array}{l}63.70(56.40 \\
73.98)\end{array}$ & $\begin{array}{l}p= \\
0.968\end{array}$ \\
\hline $\mathrm{TC}(\mathrm{mmol} / \mathrm{l})$ & $5.08(4.54,5.70)$ & $5.21(4.79,5.69)$ & $5.08(4.53,5.70)$ & $\begin{array}{l}p= \\
0.447\end{array}$ \\
\hline $\mathrm{TG}(\mathrm{mmol} / \mathrm{l})$ & $1.12(0.81,1.58)$ & $1.41(1.04,1.93)$ & $1.11(0.81,1.57)$ & $\begin{array}{l}p= \\
0.002 *\end{array}$ \\
\hline HDL-c(mmol/l) & $1.46(1.25,1.73)$ & $1.31(1.13,1.55)$ & $1.47(1.25,1.73)$ & $\begin{array}{l}\mathrm{p}< \\
0.001 *\end{array}$ \\
\hline LDL-c(mmol/l) & $3.09(2.62,3.58)$ & $3.31(3.00,3.58)$ & $3.08(2.63,3.58)$ & $\begin{array}{l}p= \\
0.063\end{array}$ \\
\hline $\mathrm{FBG}(\mathrm{mmol} / \mathrm{l})$ & $5.22(4.96,5.52)$ & $5.49(5.29,5.74)$ & $5.21(4.96,5.51)$ & $\begin{array}{l}\mathrm{p}< \\
0.001 *\end{array}$ \\
\hline PBG(mmol/l) & $6.06(5.31,6.76)$ & $6.33(5.89,6.95)$ & $6.05(5.30,6.76)$ & $\begin{array}{l}p= \\
0.014 *\end{array}$ \\
\hline
\end{tabular}

Data were mean $\pm S D$ or median (IQR) for skewed variables or numbers (proportions) for categorical variables

* indicate significance of $P$ value $(P<0.05)$

SBP: systolic blood pressure; DBP: diastolic blood pressure; GGT: gamma-glutamyl transferase; TC: total cholesterol; TG: triglycerides; HDL-C: high-density lipoprotein cholesterol; LDL-C: low-density lipoprotein cholesterol; FBG: fasting blood glucose; PBG: postprandial blood glucose; HbA1c: hemoglobin A1c. 


\begin{tabular}{|c|c|c|c|c|}
\hline & Total $(n=2568)$ & Diabetes $(n=52)$ & $\begin{array}{l}\text { Nondiabets }(n= \\
2516)\end{array}$ & $\underset{\text { value }}{p-}$ \\
\hline $\mathrm{HbA} 1 \mathrm{C}(\%)$ & $5.70(5.40,5.90)$ & $5.90(5.70,6.10)$ & $5.70(5.40,5.90)$ & $\begin{array}{l}\mathrm{p}< \\
0.001 *\end{array}$ \\
\hline \multicolumn{5}{|c|}{ Data were mean $\pm S D$ or median (IQR) for skewed variables or numbers (proportions) for categorical variables } \\
\hline \multicolumn{5}{|l|}{ * indicate significance of $P$ value $(P<0.05)$} \\
\hline $\begin{array}{l}\text { SBP: systolic blood pressure; DBP: diastolic } \\
\text { cholesterol; TG: triglycerides; HDL:C: high-de } \\
\text { FBG: fasting blood qlucose; PBG: postprand }\end{array}$ & $\begin{array}{l}\text { Dod pressure; GGT } \\
\text { ity lipoprotein chol } \\
\text { blood qlucose; } \mathrm{Hb}\end{array}$ & $\begin{array}{l}\text { amma-glutamyl tra } \\
\text { sterol; LDL-C: low-de } \\
\text { 1c: hemoglobin A1c }\end{array}$ & $\begin{array}{l}\text { Isferase; TC: total } \\
\text { isity lipoprotein ch }\end{array}$ & esterol; \\
\hline
\end{tabular}


Table 2

Baseline characteristics of study population grouped by prediabetes and normal

\begin{tabular}{|c|c|c|c|c|c|}
\hline & & Total $(n=2516)$ & $\begin{array}{l}\text { Prediabetes }(n= \\
\text { 423) }\end{array}$ & $\begin{array}{l}\text { Normal }(n= \\
2093)\end{array}$ & $\begin{array}{l}p- \\
\text { value }\end{array}$ \\
\hline Age(years old) & & $62.65 \pm 7.00$ & $64.06 \pm 6.94$ & $62.36 \pm 6.98$ & $\begin{array}{l}\mathrm{p}< \\
0.001 *\end{array}$ \\
\hline \multirow[t]{2}{*}{ Gender } & male & $790(31.40 \%)$ & $146(34.52 \%)$ & 644(30.77\%) & \multirow{2}{*}{$\begin{array}{l}p= \\
0.135\end{array}$} \\
\hline & female & $1726(68.60 \%)$ & $277(65.48 \%)$ & $1449(69.23 \%)$ & \\
\hline \multirow[t]{2}{*}{ Marital status(n, \%) } & married & 2385(94.79\%) & $404(95.51 \%)$ & $1981(94.65 \%)$ & \multirow{2}{*}{$\begin{array}{l}p= \\
0.478\end{array}$} \\
\hline & $\begin{array}{l}\text { not married } \\
\text { /widowed }\end{array}$ & $131(5.21 \%)$ & $19(4.49 \%)$ & $112(5.35 \%)$ & \\
\hline \multirow{2}{*}{$\begin{array}{l}\text { Current employment(n, } \\
\%)\end{array}$} & yes & $577(22.93 \%)$ & $66(15.60 \%)$ & $511(24.41 \%)$ & \multirow{2}{*}{$\begin{array}{l}p< \\
0.001 *\end{array}$} \\
\hline & no & 1939(77.07\%) & $357(84.40 \%)$ & $1582(75.59 \%)$ & \\
\hline \multirow[t]{5}{*}{ Education(n, \%) } & illiteracy & $16(0.64 \%)$ & $3(0.71 \%)$ & $13(0.62 \%)$ & \multirow{5}{*}{$\begin{array}{l}p= \\
0.002 *\end{array}$} \\
\hline & primary schlool & $72(2.86 \%)$ & $11(2.60 \%)$ & $61(2.91 \%)$ & \\
\hline & $\begin{array}{l}\text { junior high } \\
\text { school }\end{array}$ & $716(28.46 \%)$ & 155(36.64\%) & $561(26.81 \%)$ & \\
\hline & $\begin{array}{l}\text { senior high } \\
\text { school }\end{array}$ & $1218(48.41 \%)$ & $181(42.79 \%)$ & $1037(49.55 \%)$ & \\
\hline & college & $494(19.63 \%)$ & 73(17.26\%) & $421(20.11 \%)$ & \\
\hline \multirow[t]{2}{*}{ Current smoker(n, \%) } & yes & 459(18.24\%) & 84(19.86\%) & $375(17.92 \%)$ & \multirow{2}{*}{$\begin{array}{l}p= \\
0.369\end{array}$} \\
\hline & no & $2057(81.76 \%)$ & $339(80.14 \%)$ & $1718(82.08 \%)$ & \\
\hline \multirow{2}{*}{$\begin{array}{l}\text { Current alcohol } \\
\text { consumption(n, \%) }\end{array}$} & yes & 788(31.32\%) & $148(35.00 \%)$ & $640(30.58 \%)$ & \multirow{2}{*}{$\begin{array}{l}p= \\
0.075\end{array}$} \\
\hline & no & $1728(68.68 \%)$ & $275(65.00 \%)$ & $1453(69.42 \%)$ & \\
\hline \multirow{2}{*}{$\begin{array}{l}\text { Current tea } \\
\text { consumption(n, \%) }\end{array}$} & yes & $1609(63.95 \%)$ & $263(62.17 \%)$ & $1346(64.31 \%)$ & \multirow{2}{*}{$\begin{array}{l}p= \\
0.406\end{array}$} \\
\hline & no & $907(36.05 \%)$ & $160(37.83 \%)$ & $747(35.69 \%)$ & \\
\hline \multirow[t]{2}{*}{ Regular exercise(n, \%) } & yes & $277(11.01 \%)$ & $65(15.37 \%)$ & $212(10.13 \%)$ & \multirow{2}{*}{$\begin{array}{l}p= \\
0.002 *\end{array}$} \\
\hline & no & $2239(88.99 \%)$ & $358(84.63 \%)$ & $1881(89.87 \%)$ & \\
\hline sleeptime(h) & & $7.50(7.00,8.00)$ & $7.50(7.00,8.00)$ & $7.50(7.00,8.00)$ & $\begin{array}{l}p= \\
0.345\end{array}$ \\
\hline \multirow[t]{2}{*}{ Diabetes family history } & yes & $570(22.66 \%)$ & $116(27.42 \%)$ & $454(21.69 \%)$ & \multirow{2}{*}{$\begin{array}{l}p= \\
0.011 *\end{array}$} \\
\hline & no & 1946(77.34\%) & $307(72.58 \%)$ & 1639(78.31\%) & \\
\hline
\end{tabular}

Data were mean \pm SD or median (IQR) for skewed variables or numbers (proportions) for categorical variables

* indicate significance of $P$ value $(P<0.05)$

SBP: systolic blood pressure; DBP: diastolic blood pressure; GGT: gamma-glutamyl transferase; TC: total cholesterol; TG: triglycerides; HDL-C: high-density lipoprotein cholesterol; LDL-C: low-density lipoprotein cholesterol; FBG: fasting blood glucose; PBG: postprandial blood glucose; HbA1c: hemoglobin A1c. 


\begin{tabular}{|c|c|c|c|c|}
\hline & Total $(n=2516)$ & $\begin{array}{l}\text { Prediabetes }(n= \\
\text { 423) }\end{array}$ & $\begin{array}{l}\text { Normal }(n= \\
2093)\end{array}$ & $\begin{array}{l}p- \\
\text { value }\end{array}$ \\
\hline $\mathrm{SBP}(\mathrm{mmHg})$ & $\begin{array}{l}\text { 126.33(117.00, } \\
136.67)\end{array}$ & $\begin{array}{l}130.00(120.00 \\
140.00)\end{array}$ & $\begin{array}{l}\text { 125.67(116.33, } \\
136.00)\end{array}$ & $\begin{array}{l}\mathrm{p}< \\
0.001 *\end{array}$ \\
\hline $\mathrm{DBP}(\mathrm{mmHg})$ & $\begin{array}{l}\text { 74.67(68.33, } \\
80.67)\end{array}$ & $\begin{array}{l}\text { 76.00(70.00, } \\
81.33)\end{array}$ & $\begin{array}{l}\text { 74.33(68.00, } \\
80.67)\end{array}$ & $\begin{array}{l}\mathrm{p}= \\
0.001 *\end{array}$ \\
\hline Pulse(bp) & $\begin{array}{l}\text { 76.33(70.00, } \\
83.67)\end{array}$ & $\begin{array}{l}76.67(69.00 \\
85.00)\end{array}$ & $\begin{array}{l}\text { 76.33(70.00, } \\
83.67)\end{array}$ & $\begin{array}{l}p= \\
0.573\end{array}$ \\
\hline WHtR & $0.50(0.47,0.53)$ & $\begin{array}{l}0.511(0.48 \\
0.54)\end{array}$ & $0.50(0.46,0.53)$ & $\begin{array}{l}\mathrm{p}< \\
0.001 *\end{array}$ \\
\hline Height(cm) & $\begin{array}{l}\text { 161.00(156.00, } \\
167.00)\end{array}$ & $\begin{array}{l}\text { 160.50(155.80, } \\
16.50)\end{array}$ & $\begin{array}{l}\text { 161.00(156.00, } \\
167.00)\end{array}$ & $\begin{array}{l}p= \\
0.598\end{array}$ \\
\hline Weight(kg) & $\begin{array}{l}64.00(58.00 \\
72.00)\end{array}$ & $\begin{array}{l}66.00(60.00 \\
74.00)\end{array}$ & $\begin{array}{l}\text { 64.00(57.00, } \\
71.50)\end{array}$ & $\begin{array}{l}p< \\
0.001 *\end{array}$ \\
\hline BMI & $\begin{array}{l}24.76(22.74, \\
27.04)\end{array}$ & $\begin{array}{l}25.83(23.57 \\
27.82)\end{array}$ & $\begin{array}{l}24.61(22.60 \\
26.82)\end{array}$ & $\begin{array}{l}\mathrm{p}< \\
0.001 *\end{array}$ \\
\hline WC(cm) & $\begin{array}{l}80.55(75.00 \\
86.00)\end{array}$ & $\begin{array}{l}\text { 83.00(78.00, } \\
89.00)\end{array}$ & $\begin{array}{l}\text { 80.00(74.00, } \\
86.00)\end{array}$ & $\begin{array}{l}\mathrm{p}< \\
0.001 *\end{array}$ \\
\hline $\mathrm{HC}(\mathrm{cm})$ & $\begin{array}{l}92.00(89.00, \\
96.50)\end{array}$ & $\begin{array}{l}93.00(89.00, \\
97.00)\end{array}$ & $\begin{array}{l}92.00(88.50, \\
96.00)\end{array}$ & $\begin{array}{l}\mathrm{p}= \\
0.016 *\end{array}$ \\
\hline WHR & $0.87(0.83,0.91)$ & $0.89(0.85,0.93)$ & $0.87(0.83,0.91)$ & $\begin{array}{l}\mathrm{p}< \\
0.001 *\end{array}$ \\
\hline GGT & $\begin{array}{l}17.60(13.50 \\
24.80)\end{array}$ & $\begin{array}{l}19.40(14.40 \\
27.00)\end{array}$ & $\begin{array}{l}\text { 17.30(13.40, } \\
24.25)\end{array}$ & $\begin{array}{l}\mathrm{p}< \\
0.001 *\end{array}$ \\
\hline $\mathrm{Cr}$ & $\begin{array}{l}\text { 63.70(56.40, } \\
73.98)\end{array}$ & $\begin{array}{l}65.00(56.40 \\
75.30)\end{array}$ & $\begin{array}{l}\text { 63.50(56.40, } \\
73.70)\end{array}$ & $\begin{array}{l}p= \\
0.135\end{array}$ \\
\hline $\mathrm{TC}(\mathrm{mmol} / \mathrm{l})$ & $5.08(4.53,5.70)$ & $5.15(4.64,5.78)$ & $5.06(4.51,5.69)$ & $\begin{array}{l}p= \\
0.058\end{array}$ \\
\hline $\mathrm{TG}(\mathrm{mmol} / \mathrm{l})$ & $1.11(0.81,1.57)$ & $1.21(0.86,1.81)$ & $1.09(0.80,1.52)$ & $\begin{array}{l}\mathrm{p}< \\
0.001 *\end{array}$ \\
\hline $\mathrm{HDL}-\mathrm{c}(\mathrm{mmol} / \mathrm{l})$ & $1.47(1.25,1.73)$ & $1.41(1.22,1.68)$ & $1.48(1.26,1.74)$ & $\begin{array}{l}\mathrm{p}= \\
0.002^{*}\end{array}$ \\
\hline LDL-c(mmol/l) & $3.08(2.62,3.58)$ & $3.16(2.69,3.69)$ & $3.06(2.60,3.54)$ & $\begin{array}{l}p= \\
0.008 *\end{array}$ \\
\hline $\mathrm{FBG}(\mathrm{mmol} / \mathrm{l})$ & $5.21(4.96,5.51)$ & $5.42(5.11,5.74)$ & $5.18(4.94,5.45)$ & $\begin{array}{l}\mathrm{p}< \\
0.001 *\end{array}$ \\
\hline PBG(mmol/l) & $6.06(5.30,6.76)$ & $6.44(5.57,6.99)$ & $5.98(5.24,6.70)$ & $\begin{array}{l}p< \\
0.001 *\end{array}$ \\
\hline
\end{tabular}

Data were mean $\pm S D$ or median (IQR) for skewed variables or numbers (proportions) for categorical variables

* indicate significance of $P$ value $(P<0.05)$

SBP: systolic blood pressure; DBP: diastolic blood pressure; GGT: gamma-glutamyl transferase; TC: total cholesterol; TG: triglycerides; HDL-C: high-density lipoprotein cholesterol; LDL-C: low-density lipoprotein cholesterol; FBG: fasting blood glucose; PBG: postprandial blood glucose; HbA1c: hemoglobin A1c. 


\begin{tabular}{|c|c|c|c|c|}
\hline & Total $(n=2516)$ & $\begin{array}{l}\text { Prediabetes }(n= \\
423)\end{array}$ & $\begin{array}{l}\text { Normal }(n= \\
2093)\end{array}$ & $\begin{array}{l}p- \\
\text { value }\end{array}$ \\
\hline HbA1C(\%) & $5.70(5.40,5.90$ & $5.80(5.60,6.00)$ & $5.70(5.40,5.90)$ & $\begin{array}{l}p< \\
0.001 *\end{array}$ \\
\hline
\end{tabular}

Data were mean \pm SD or median (IQR) for skewed variables or numbers (proportions) for categorical variables

* indicate significance of $P$ value $(P<0.05)$

SBP: systolic blood pressure; DBP: diastolic blood pressure; GGT: gamma-glutamyl transferase; TC: total cholesterol; TG: triglycerides; HDL-C: high-density lipoprotein cholesterol; LDL-C: low-density lipoprotein cholesterol; FBG: fasting blood glucose; PBG: postprandial blood glucose; HbA1c: hemoglobin A1c.

\section{Association of anthropometric values with incident diabetes risk}

To further analyze the function of these anthropometrics, we used stratified analyses for different sexes. To better discuss the relationship, we divided the WHtR, BMI, WC and WHR into tertiles. The results are shown in Table 3. Although all of these anthropometric values are meaningful in the general population, the results change after stratification by gender. The results showed that these 4 indices were significantly different between diabetic and nondiabetic participants in the female group, but the difference disappeared in the male group. After adjusting for age in the general population, a difference also existed in Tertile 3, while no significant difference was observed in the multivariate-adjusted model. A difference in BMI existed in the age-adjusted model in both female and male (female: Tertile 3, OR: 2.88, 95\% Cl: 1.13-7.33, $p=0.026$; male: Tertile 2, OR: 5.04, 95\% Cl: 1.09-23.25, $p=0.038$ ), while this difference disappeared in the multivariate-adjusted model. No differences were found in other anthropometric values. 
Table 3

Age-adjusted and multivariable association of different anthropometric indices with the risk of diabetes

\begin{tabular}{|c|c|c|c|c|c|c|c|c|c|}
\hline & & & \multirow{2}{*}{$\begin{array}{l}\text { Diabetes } \\
\% \\
\text { (n/total) }\end{array}$} & \multirow[t]{2}{*}{$\begin{array}{l}\text { Nondiabetes } \\
\%(n / \text { total) }\end{array}$} & \multirow[t]{2}{*}{$\begin{array}{l}p- \\
\text { value }\end{array}$} & \multicolumn{2}{|c|}{ Age-adjusted } & \multicolumn{2}{|c|}{$\begin{array}{l}\text { Multivariate- } \\
\text { adjusted }\end{array}$} \\
\hline & & & & & & $\begin{array}{l}\text { OR(95\% } \\
\mathrm{Cl})\end{array}$ & $\begin{array}{l}p- \\
\text { value }\end{array}$ & $\begin{array}{l}\text { OR(95\% } \\
\mathrm{Cl})\end{array}$ & $\begin{array}{l}\mathrm{p}- \\
\text { value }\end{array}$ \\
\hline \multirow[t]{12}{*}{$\begin{array}{l}\text { Tota(n = } \\
2568)\end{array}$} & \multirow[t]{3}{*}{ WHtR } & $\begin{array}{l}\text { Tertile } \\
1\end{array}$ & $\begin{array}{l}19.23 \% \\
(10 / 52)\end{array}$ & $\begin{array}{l}33.87 \% \\
(852 / 2516)\end{array}$ & \multirow[t]{3}{*}{$\begin{array}{l}p= \\
0.019 *\end{array}$} & reference & & \multicolumn{2}{|l|}{ reference } \\
\hline & & $\begin{array}{l}\text { Tertile } \\
2\end{array}$ & $\begin{array}{l}30.77 \% \\
(16 / 52)\end{array}$ & $\begin{array}{l}33.30 \% \\
(838 / 2516)\end{array}$ & & $\begin{array}{l}1.59(0.72 \\
3.53)\end{array}$ & $\begin{array}{l}p= \\
0.256\end{array}$ & $\begin{array}{l}1.24(0.55 \\
2.82)\end{array}$ & $p=0.6$ \\
\hline & & $\begin{array}{l}\text { Tertile } \\
3\end{array}$ & $\begin{array}{l}50 \% \\
(26 / 52)\end{array}$ & $\begin{array}{l}32.83 \% \\
(826 / 2516)\end{array}$ & & $\begin{array}{l}2.53(1.20 \\
5.34)\end{array}$ & $\begin{array}{l}p= \\
0.014 *\end{array}$ & $\begin{array}{l}1.56(0.71 \\
3.40)\end{array}$ & $\begin{array}{l}p= \\
0.27\end{array}$ \\
\hline & \multirow[t]{3}{*}{ BMI } & $\begin{array}{l}\text { Tertile } \\
1\end{array}$ & $\begin{array}{l}19.23 \% \\
(10 / 52)\end{array}$ & $\begin{array}{l}33.74 \% \\
(849 / 2516)\end{array}$ & \multirow[t]{3}{*}{$\begin{array}{l}p= \\
0.01 *\end{array}$} & reference & & \multicolumn{2}{|l|}{ reference } \\
\hline & & $\begin{array}{l}\text { Tertile } \\
2\end{array}$ & $\begin{array}{l}28.85 \% \\
(15 / 52)\end{array}$ & $\begin{array}{l}33.30 \% \\
(838 / 2516)\end{array}$ & & $\begin{array}{l}1.50(0.67 \\
3.35)\end{array}$ & $\begin{array}{l}p= \\
0.327\end{array}$ & $\begin{array}{l}1.17(0.51 \\
2.67)\end{array}$ & $\begin{array}{l}\mathrm{p}= \\
0.72\end{array}$ \\
\hline & & $\begin{array}{l}\text { Tertile } \\
3\end{array}$ & $\begin{array}{l}51.92 \% \\
(27 / 52)\end{array}$ & $\begin{array}{l}32.96 \% \\
(829 / 2516)\end{array}$ & & $\begin{array}{l}2.71(1.30 \\
5.64)\end{array}$ & $\begin{array}{l}p= \\
0.008 *\end{array}$ & $\begin{array}{l}1.50(0.69 \\
3.28)\end{array}$ & $\begin{array}{l}p= \\
0.31\end{array}$ \\
\hline & \multirow[t]{3}{*}{ WC } & $\begin{array}{l}\text { Tertile } \\
1\end{array}$ & $\begin{array}{l}17.31 \% \\
(9 / 52)\end{array}$ & $\begin{array}{l}35.10 \% \\
(883 / 2516)\end{array}$ & \multirow[t]{3}{*}{$\begin{array}{l}p= \\
0.003 *\end{array}$} & reference & & \multicolumn{2}{|l|}{ reference } \\
\hline & & $\begin{array}{l}\text { Tertile } \\
2\end{array}$ & $\begin{array}{l}30.77 \% \\
(16 / 52)\end{array}$ & $\begin{array}{l}33.46 \% \\
(842 / 2516)\end{array}$ & & $\begin{array}{l}1.80(0.79 \\
4.12)\end{array}$ & $\begin{array}{l}p= \\
0.162\end{array}$ & $\begin{array}{l}1.44(0.62 \\
3.37)\end{array}$ & $\begin{array}{l}p= \\
0.39\end{array}$ \\
\hline & & $\begin{array}{l}\text { Tertile } \\
3\end{array}$ & $\begin{array}{l}51.92 \% \\
(27 / 52)\end{array}$ & $\begin{array}{l}31.44 \% \\
(791 / 2516)\end{array}$ & & $\begin{array}{l}3.17(1.47, \\
6.86)\end{array}$ & $\begin{array}{l}p= \\
0.003 *\end{array}$ & $\begin{array}{l}1.96(0.86 \\
4.49)\end{array}$ & $\begin{array}{l}p= \\
0.11\end{array}$ \\
\hline & \multirow[t]{3}{*}{ WHR } & $\begin{array}{l}\text { Tertile } \\
1\end{array}$ & $\begin{array}{l}17.31 \% \\
(9 / 52)\end{array}$ & $\begin{array}{l}33.74 \% \\
(849 / 2516)\end{array}$ & \multirow[t]{3}{*}{$\begin{array}{l}p= \\
0.01 *\end{array}$} & reference & & \multicolumn{2}{|l|}{ reference } \\
\hline & & $\begin{array}{l}\text { Tertile } \\
2\end{array}$ & $\begin{array}{l}32.69 \% \\
(17 / 52)\end{array}$ & $\begin{array}{l}34.18 \% \\
(860 / 2516)\end{array}$ & & $\begin{array}{l}1.81(0.80 \\
4.09)\end{array}$ & $\begin{array}{l}p= \\
0.156\end{array}$ & $\begin{array}{l}1.23(0.53 \\
2.85)\end{array}$ & $\begin{array}{l}p= \\
0.627\end{array}$ \\
\hline & & $\begin{array}{l}\text { Tertile } \\
3\end{array}$ & $\begin{array}{l}50 \% \\
(26 / 52)\end{array}$ & $\begin{array}{l}32.08 \% \\
(807 / 2516)\end{array}$ & & $\begin{array}{l}2.86(1.32, \\
6.23)\end{array}$ & $\begin{array}{l}p= \\
0.008 *\end{array}$ & $\begin{array}{l}1.53(0.67 \\
3.48)\end{array}$ & $\begin{array}{l}p= \\
0.316\end{array}$ \\
\hline \multirow[t]{5}{*}{$\begin{array}{l}\text { Female(n } \\
=1758)\end{array}$} & \multirow[t]{3}{*}{ WHtR } & $\begin{array}{l}\text { Tertile } \\
1\end{array}$ & $\begin{array}{l}21.88 \% \\
(7 / 32)\end{array}$ & $\begin{array}{l}33.66 \% \\
(581 / 1726)\end{array}$ & \multirow[t]{3}{*}{$\begin{array}{l}p= \\
0.023 *\end{array}$} & reference & & \multicolumn{2}{|l|}{ reference } \\
\hline & & $\begin{array}{l}\text { Tertile } \\
2\end{array}$ & $\begin{array}{l}21.88 \% \\
(7 / 32)\end{array}$ & $\begin{array}{l}33.49 \% \\
(578 / 1726)\end{array}$ & & $\begin{array}{l}0.96(0.34, \\
2.77)\end{array}$ & $\begin{array}{l}p= \\
0.946\end{array}$ & $\begin{array}{l}0.73(0.25 \\
2.15)\end{array}$ & $\begin{array}{l}p= \\
0.569\end{array}$ \\
\hline & & $\begin{array}{l}\text { Tertile } \\
3\end{array}$ & $\begin{array}{l}56.24 \% \\
(18 / 32)\end{array}$ & $\begin{array}{l}32.85 \% \\
(567 / 1726)\end{array}$ & & $\begin{array}{l}2.26(0.91 \\
5.60)\end{array}$ & $\begin{array}{l}p= \\
0.079\end{array}$ & $\begin{array}{l}1.40(0.54 \\
3.68)\end{array}$ & $\begin{array}{l}p= \\
0.49\end{array}$ \\
\hline & \multirow[t]{2}{*}{$\mathrm{BMI}$} & $\begin{array}{l}\text { Tertile } \\
1\end{array}$ & $\begin{array}{l}18.75 \% \\
(6 / 32)\end{array}$ & $\begin{array}{l}33.55 \% \\
(579 / 1726)\end{array}$ & \multirow[t]{2}{*}{$\begin{array}{l}p= \\
0.021 *\end{array}$} & reference & & \multicolumn{2}{|l|}{ reference } \\
\hline & & $\begin{array}{l}\text { Tertile } \\
2\end{array}$ & $\begin{array}{l}25 \% \\
(8 / 32)\end{array}$ & $\begin{array}{l}33.49 \% \\
(578 / 1726)\end{array}$ & & $\begin{array}{l}\text { 1.32(0.46, } \\
3.83)\end{array}$ & $\begin{array}{l}p= \\
0.609\end{array}$ & $\begin{array}{l}0.99(0.34, \\
2.94)\end{array}$ & $\begin{array}{l}\mathrm{p}= \\
0.989\end{array}$ \\
\hline
\end{tabular}

Data were mean \pm SD or median (IQR) for skewed variables or numbers (proportions) for categorical variables

* indicate significance of $P$ value $(P<0.05)$

Multivariable Logistic models to adjust for the following confounding factors: age, SBP, diabetes family history, GGT, TG, HDL-c and HbA1C. 


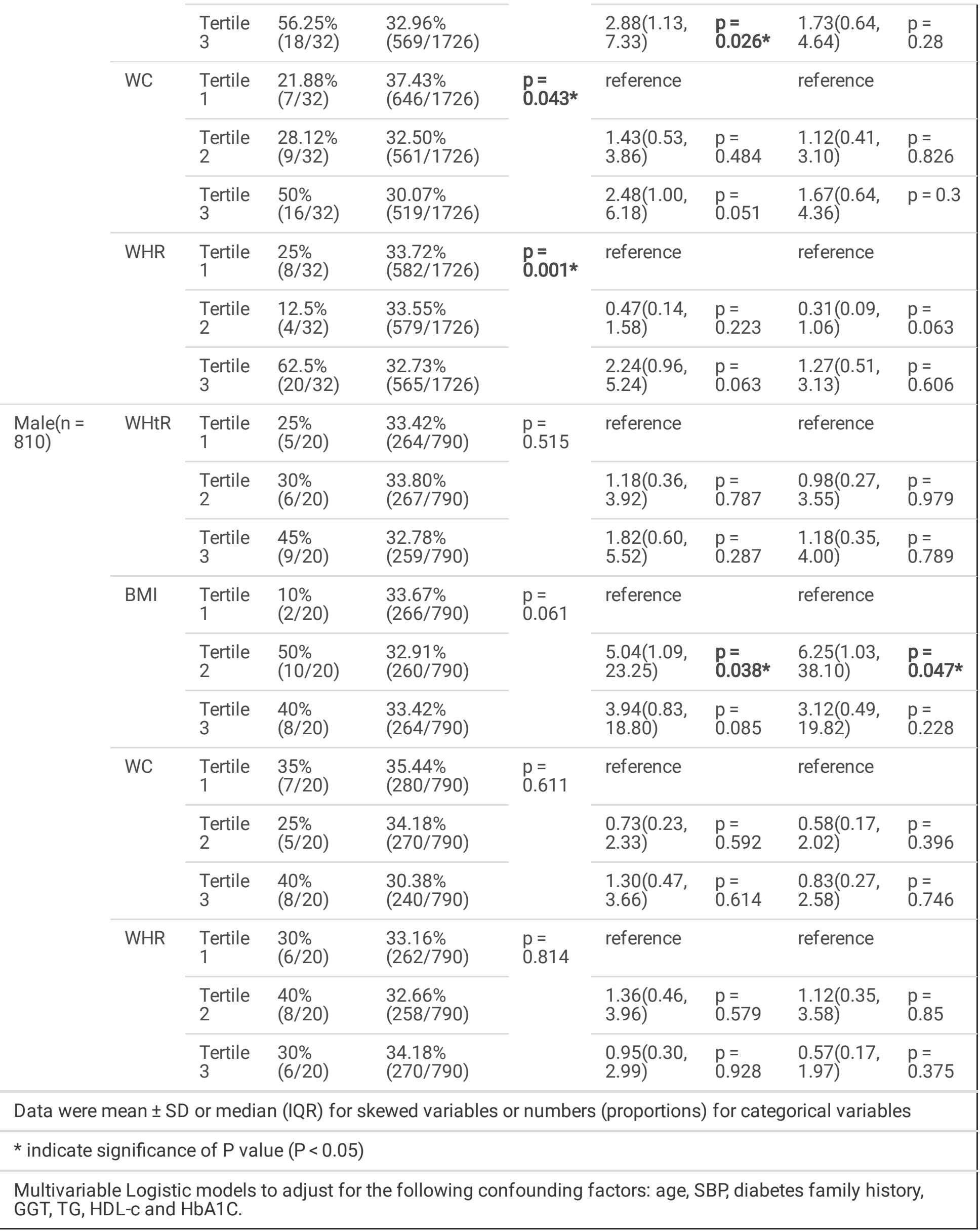




\section{Association of different anthropometric values with incident prediabetes risk}

In order to better discuss the relationship between different anthropometric values and blood glucose status, we further divided people into prediabetic and normal blood glucose groups. The results are shown in Table 4. The age-adjusted model in the general population showed that the WHtR has the highest OR (Tertile 2 OR: 1.85, 95\% Cl 1.39-2.45, p< 0.001; Tertile 3 OR: 2.25, 95\% Cl: 1.68-2.94, p < 0.001) compared with other values, which means that people with a higher WHtR have a higher risk of prediabetes. Moreover, as the WHtR increased, the OR increased. This phenomenon was also true in the multivariate-adjusted model (Tertile 2 OR: 1.72, 95\% Cl 1.28-2.30, p< 0.001; Tertile 3 OR: 1.90, 95\% $\mathrm{Cl}: 1.41-2.56, \mathrm{p}<0.001)$. The association between the WHtR and prediabetes was the strongest among all four indices. To summarize, these four indices in the prediabetes group tended to follow the following pattern: WHtR $<$ WC $<$ WHR $<$ BMI. 
Table 4

Age-adjusted and multivariable association of different anthropometric indices with the risk of prediabetes

\begin{tabular}{|c|c|c|c|c|c|c|c|c|c|}
\hline & & & \multirow[t]{2}{*}{$\begin{array}{l}\text { Prediabetes } \\
\%(\text { n/total) }\end{array}$} & \multirow[t]{2}{*}{$\begin{array}{l}\text { Normal \% } \\
\text { (n/total) }\end{array}$} & \multirow[t]{2}{*}{$\begin{array}{l}p- \\
\text { value }\end{array}$} & \multicolumn{2}{|c|}{ Age-adjusted } & \multicolumn{2}{|c|}{$\begin{array}{l}\text { Multivariate- } \\
\text { adjusted }\end{array}$} \\
\hline & & & & & & $\begin{array}{l}\text { OR(95\% } \\
\mathrm{Cl})\end{array}$ & $\begin{array}{l}\mathrm{p} \text { - } \\
\text { value }\end{array}$ & $\begin{array}{l}\text { OR(95\% } \\
\mathrm{Cl})\end{array}$ & $\begin{array}{l}\mathrm{p}- \\
\text { value }\end{array}$ \\
\hline \multirow[t]{12}{*}{$\begin{array}{l}\text { Tota }(\mathrm{n}= \\
2516)\end{array}$} & \multirow[t]{3}{*}{$\mathrm{WHtR}$} & $\begin{array}{l}\text { Tertile } \\
1\end{array}$ & $\begin{array}{l}20.80 \% \\
(88 / 423)\end{array}$ & $\begin{array}{l}36.12 \% \\
(756 / 2093)\end{array}$ & $\begin{array}{l}p< \\
0.001 *\end{array}$ & reference & & reference & \\
\hline & & $\begin{array}{l}\text { Tertile } \\
2\end{array}$ & $\begin{array}{l}35.70 \% \\
(151 / 423)\end{array}$ & $\begin{array}{l}32.54 \% \\
(681 / 2093)\end{array}$ & & $\begin{array}{l}1.85(1.39 \\
2.45)\end{array}$ & $\begin{array}{l}p< \\
0.001 *\end{array}$ & $\begin{array}{l}1.72(1.28, \\
2.30)\end{array}$ & $\begin{array}{l}p< \\
0.001 *\end{array}$ \\
\hline & & $\begin{array}{l}\text { Tertile } \\
3\end{array}$ & $\begin{array}{l}43.50 \% \\
(184 / 423)\end{array}$ & $\begin{array}{l}31.34 \% \\
(656 / 2093)\end{array}$ & & $\begin{array}{l}2.25(1.68, \\
2.94)\end{array}$ & $\begin{array}{l}p< \\
0.001 *\end{array}$ & $\begin{array}{l}1.90(1.41, \\
2.56)\end{array}$ & $\begin{array}{l}\mathrm{p}< \\
0.001 *\end{array}$ \\
\hline & \multirow[t]{3}{*}{ BMI } & $\begin{array}{l}\text { Tertile } \\
1\end{array}$ & $\begin{array}{l}23.40 \% \\
(99 / 423)\end{array}$ & $\begin{array}{l}35.64 \% \\
(746 / 2093)\end{array}$ & $\begin{array}{l}p< \\
0.001 *\end{array}$ & reference & & reference & \\
\hline & & $\begin{array}{l}\text { Tertile } \\
2\end{array}$ & $\begin{array}{l}32.39 \% \\
(137 / 423)\end{array}$ & $\begin{array}{l}33.16 \% \\
(694 / 2093)\end{array}$ & & $\begin{array}{l}1.46(1.11, \\
1.93)\end{array}$ & $\begin{array}{l}p= \\
0.008 *\end{array}$ & $\begin{array}{l}1.33(1.00 \\
1.77)\end{array}$ & $\begin{array}{l}p= \\
0.053\end{array}$ \\
\hline & & $\begin{array}{l}\text { Tertile } \\
3\end{array}$ & $\begin{array}{l}44.21 \% \\
(187 / 423)\end{array}$ & $\begin{array}{l}31.20 \% \\
(653 / 2093)\end{array}$ & & $\begin{array}{l}2.11(1.62, \\
2.76)\end{array}$ & $\begin{array}{l}p< \\
0.001 *\end{array}$ & $\begin{array}{l}1.65(1.23 \\
2.21)\end{array}$ & $\begin{array}{l}p< \\
0.001 *\end{array}$ \\
\hline & \multirow[t]{3}{*}{ WC } & $\begin{array}{l}\text { Tertile } \\
1\end{array}$ & $\begin{array}{l}24.11 \% \\
(102 / 423)\end{array}$ & $\begin{array}{l}37.31 \% \\
(781 / 2093)\end{array}$ & $\begin{array}{l}p< \\
0.001 *\end{array}$ & reference & & reference & \\
\hline & & $\begin{array}{l}\text { Tertile } \\
2\end{array}$ & $\begin{array}{l}35.70 \% \\
(151 / 423)\end{array}$ & $\begin{array}{l}33.02 \% \\
(691 / 2093)\end{array}$ & & $\begin{array}{l}1.59(1.21, \\
2.09)\end{array}$ & $\begin{array}{l}p= \\
0.001 *\end{array}$ & $\begin{array}{l}1.53(1.15 \\
2.03)\end{array}$ & $\begin{array}{l}p= \\
0.003 *\end{array}$ \\
\hline & & $\begin{array}{l}\text { Tertile } \\
3\end{array}$ & $\begin{array}{l}40.19 \% \\
(170 / 423)\end{array}$ & $\begin{array}{l}29.67 \% \\
(621 / 2093)\end{array}$ & & $\begin{array}{l}1.92(1.47 \\
2.52)\end{array}$ & $\begin{array}{l}\mathrm{p}< \\
0.001 *\end{array}$ & $\begin{array}{l}1.69(1.25 \\
2.29)\end{array}$ & $\begin{array}{l}p= \\
0.001 *\end{array}$ \\
\hline & \multirow[t]{3}{*}{ WHR } & $\begin{array}{l}\text { Tertile } \\
1\end{array}$ & $\begin{array}{l}22.93 \% \\
(97 / 423)\end{array}$ & $\begin{array}{l}35.16 \% \\
(736 / 2093)\end{array}$ & $\begin{array}{l}p< \\
0.001 *\end{array}$ & reference & & reference & \\
\hline & & $\begin{array}{l}\text { Tertile } \\
2\end{array}$ & $\begin{array}{l}35.93 \% \\
(152 / 423)\end{array}$ & $\begin{array}{l}34.59 \% \\
(724 / 2093)\end{array}$ & & $\begin{array}{l}1.52(1.15 \\
2.00)\end{array}$ & $\begin{array}{l}p= \\
0.003^{*}\end{array}$ & $\begin{array}{l}1.35(1.01 \\
1.79)\end{array}$ & $\begin{array}{l}p= \\
0.042 *\end{array}$ \\
\hline & & $\begin{array}{l}\text { Tertile } \\
3\end{array}$ & $\begin{array}{l}41.13 \% \\
(174 / 423)\end{array}$ & $\begin{array}{l}30.24 \% \\
(633 / 2093)\end{array}$ & & $\begin{array}{l}1.89(1.44, \\
2.50)\end{array}$ & $\begin{array}{l}\mathrm{p}< \\
0.001 *\end{array}$ & $\begin{array}{l}1.53(1.13 \\
2.06)\end{array}$ & $\begin{array}{l}p= \\
0.006 *\end{array}$ \\
\hline \multirow[t]{5}{*}{$\begin{array}{l}\text { Female(n } \\
=1726)\end{array}$} & \multirow[t]{3}{*}{$\mathrm{WHtR}$} & $\begin{array}{l}\text { Tertile } \\
1\end{array}$ & $\begin{array}{l}19.86 \% \\
(55 / 277)\end{array}$ & $\begin{array}{l}36.03 \% \\
(522 / 1449)\end{array}$ & $\begin{array}{l}\mathrm{p}< \\
0.001 *\end{array}$ & reference & & reference & \\
\hline & & $\begin{array}{l}\text { Tertile } \\
2\end{array}$ & $\begin{array}{l}35.38 \% \\
(98 / 277)\end{array}$ & $\begin{array}{l}32.78 \% \\
(475 / 1449)\end{array}$ & & $\begin{array}{l}1.90(1.34, \\
2.71)\end{array}$ & $\begin{array}{l}p< \\
0.001 *\end{array}$ & $\begin{array}{l}1.74(1.21 \\
2.51)\end{array}$ & $\begin{array}{l}p= \\
0.003 *\end{array}$ \\
\hline & & $\begin{array}{l}\text { Tertile } \\
3\end{array}$ & $\begin{array}{l}44.76 \% \\
(124 / 277)\end{array}$ & $\begin{array}{l}31.19 \% \\
(452 / 1449)\end{array}$ & & $\begin{array}{l}2.34(1.65, \\
3.33)\end{array}$ & $\begin{array}{l}p< \\
0.001 *\end{array}$ & $\begin{array}{l}2.02(1.38, \\
2.95)\end{array}$ & $\begin{array}{l}p< \\
0.001 *\end{array}$ \\
\hline & \multirow[t]{2}{*}{ BMI } & $\begin{array}{l}\text { Tertile } \\
1\end{array}$ & $\begin{array}{l}20.94 \% \\
(58 / 277)\end{array}$ & $\begin{array}{l}35.96 \% \\
(521 / 1449)\end{array}$ & $\begin{array}{l}\mathrm{p}< \\
0.001 *\end{array}$ & reference & & reference & \\
\hline & & $\begin{array}{l}\text { Tertile } \\
2\end{array}$ & $\begin{array}{l}35.38 \% \\
(98 / 277)\end{array}$ & $\begin{array}{l}32.78 \% \\
(475 / 1449)\end{array}$ & & $\begin{array}{l}1.84(1.30 \\
2.61)\end{array}$ & $\begin{array}{l}p= \\
0.001 *\end{array}$ & $\begin{array}{l}1.65(1.15, \\
2.37)\end{array}$ & $\begin{array}{l}p= \\
0.006 *\end{array}$ \\
\hline
\end{tabular}

Data were mean \pm SD or median (IQR) for skewed variables or numbers (proportions) for categorical variables

* indicate significance of $P$ value $(P<0.05)$

Multivariable Logistic models to adjust for the following confounding factors: age, employment, education, exercise, diabetes family history, SBP, DBP, GGT, TG, HDL-c and HbA1C. 


\begin{tabular}{|c|c|c|c|c|c|c|c|c|c|}
\hline & & $\begin{array}{l}\text { Tertile } \\
3\end{array}$ & $\begin{array}{l}43.68 \% \\
(121 / 277)\end{array}$ & $\begin{array}{l}31.26 \% \\
(453 / 1449)\end{array}$ & & $\begin{array}{l}2.31(1.64, \\
3.24)\end{array}$ & $\begin{array}{l}p< \\
0.001 *\end{array}$ & $\begin{array}{l}1.81(1.25 \\
2.61)\end{array}$ & $\begin{array}{l}p= \\
0.002 *\end{array}$ \\
\hline & \multirow[t]{3}{*}{ WC } & $\begin{array}{l}\text { Tertile } \\
1\end{array}$ & $\begin{array}{l}25.63 \% \\
(71 / 277)\end{array}$ & $\begin{array}{l}39.68 \% \\
(575 / 1449)\end{array}$ & $\begin{array}{l}p< \\
0.001 *\end{array}$ & reference & & reference & \\
\hline & & $\begin{array}{l}\text { Tertile } \\
2\end{array}$ & $\begin{array}{l}35.02 \% \\
(97 / 277)\end{array}$ & $\begin{array}{l}32.02 \% \\
(464 / 1449)\end{array}$ & & $\begin{array}{l}1.65(1.19, \\
2.30)\end{array}$ & $\begin{array}{l}p= \\
0.003 *\end{array}$ & $\begin{array}{l}1.53(1.09 \\
2.15)\end{array}$ & $\begin{array}{l}p= \\
0.015^{*}\end{array}$ \\
\hline & & $\begin{array}{l}\text { Tertile } \\
3\end{array}$ & $\begin{array}{l}39.35 \% \\
(109 / 277)\end{array}$ & $\begin{array}{l}28.30 \% \\
(410 / 1449)\end{array}$ & & $\begin{array}{l}1.95(1.40 \\
2.72)\end{array}$ & $\begin{array}{l}\mathrm{p}< \\
0.001 *\end{array}$ & $\begin{array}{l}1.69(1.18, \\
2.42)\end{array}$ & $\begin{array}{l}p= \\
0.004 *\end{array}$ \\
\hline & \multirow[t]{3}{*}{ WHR } & $\begin{array}{l}\text { Tertile } \\
1\end{array}$ & $\begin{array}{l}23.83 \% \\
(66 / 277)\end{array}$ & $\begin{array}{l}35.61 \% \\
(516 / 1449)\end{array}$ & $\begin{array}{l}p= \\
0.001 *\end{array}$ & reference & & reference & \\
\hline & & $\begin{array}{l}\text { Tertile } \\
2\end{array}$ & $\begin{array}{l}37.55 \% \\
(104 / 277)\end{array}$ & $\begin{array}{l}32.51 \% \\
(471 / 1449)\end{array}$ & & $\begin{array}{l}1.65(1.18, \\
2.30)\end{array}$ & $\begin{array}{l}p= \\
0.004 *\end{array}$ & $\begin{array}{l}1.46(1.03 \\
2.07)\end{array}$ & $\begin{array}{l}p= \\
0.035^{*}\end{array}$ \\
\hline & & $\begin{array}{l}\text { Tertile } \\
3\end{array}$ & $\begin{array}{l}38.63 \% \\
(107 / 277)\end{array}$ & $\begin{array}{l}31.88 \% \\
(462 / 1449)\end{array}$ & & $\begin{array}{l}1.62(1.15 \\
2.27)\end{array}$ & $\begin{array}{l}p= \\
0.005^{*}\end{array}$ & $\begin{array}{l}1.26(0.88 \\
1.81)\end{array}$ & $\begin{array}{l}p= \\
0.205\end{array}$ \\
\hline \multirow[t]{12}{*}{$\begin{array}{l}\text { Male }(n= \\
790)\end{array}$} & \multirow[t]{3}{*}{$\mathrm{WHtR}$} & $\begin{array}{l}\text { Tertile } \\
1\end{array}$ & $\begin{array}{l}23.97 \% \\
(35 / 146)\end{array}$ & $\begin{array}{l}35.56 \% \\
(229 / 644)\end{array}$ & $\begin{array}{l}p= \\
0.021 *\end{array}$ & reference & & reference & \\
\hline & & $\begin{array}{l}\text { Tertile } \\
2\end{array}$ & $\begin{array}{l}35.62 \% \\
(52 / 146)\end{array}$ & $\begin{array}{l}32.76 \% \\
(211 / 644)\end{array}$ & & $\begin{array}{l}1.63(1.02 \\
2.60)\end{array}$ & $\begin{array}{l}\mathrm{p}= \\
0.042^{*}\end{array}$ & $\begin{array}{l}1.55(0.95 \\
2.52)\end{array}$ & $\begin{array}{l}p= \\
0.08\end{array}$ \\
\hline & & $\begin{array}{l}\text { Tertile } \\
3\end{array}$ & $\begin{array}{l}40.41 \% \\
(59 / 146)\end{array}$ & $\begin{array}{l}31.68 \% \\
(204 / 644)\end{array}$ & & $\begin{array}{l}1.90(1.20 \\
3.02)\end{array}$ & $\begin{array}{l}p= \\
0.006 *\end{array}$ & $\begin{array}{l}1.57(0.96 \\
2.59)\end{array}$ & $\begin{array}{l}p= \\
0.074\end{array}$ \\
\hline & \multirow[t]{3}{*}{ BMI } & $\begin{array}{l}\text { Tertile } \\
1\end{array}$ & $\begin{array}{l}26.71 \% \\
(39 / 146)\end{array}$ & $\begin{array}{l}34.94 \% \\
(225 / 644)\end{array}$ & $\begin{array}{l}p= \\
0.004^{*}\end{array}$ & reference & & reference & \\
\hline & & $\begin{array}{l}\text { Tertile } \\
2\end{array}$ & $\begin{array}{l}28.08 \% \\
(41 / 146)\end{array}$ & $\begin{array}{l}34.32 \% \\
(221 / 644)\end{array}$ & & $\begin{array}{l}1.11(0.69 \\
1.79)\end{array}$ & $\begin{array}{l}p= \\
0.679\end{array}$ & $\begin{array}{l}1.00(0.62 \\
1.64)\end{array}$ & $\begin{array}{l}p= \\
0.991\end{array}$ \\
\hline & & $\begin{array}{l}\text { Tertile } \\
3\end{array}$ & $\begin{array}{l}45.21 \% \\
(66 / 146)\end{array}$ & $\begin{array}{l}30.74 \% \\
(198 / 644)\end{array}$ & & $\begin{array}{l}2.12(1.29 \\
3.14)\end{array}$ & $\begin{array}{l}p= \\
0.002 *\end{array}$ & $\begin{array}{l}1.55(0.96 \\
2.51)\end{array}$ & $\begin{array}{l}p= \\
0.075\end{array}$ \\
\hline & \multirow[t]{3}{*}{ WC } & $\begin{array}{l}\text { Tertile } \\
1\end{array}$ & $\begin{array}{l}28.08 \% \\
(41 / 146)\end{array}$ & $\begin{array}{l}37.10 \% \\
(239 / 644)\end{array}$ & $\begin{array}{l}p= \\
0.071\end{array}$ & reference & & reference & \\
\hline & & $\begin{array}{l}\text { Tertile } \\
2\end{array}$ & $\begin{array}{l}34.93 \% \\
(51 / 146)\end{array}$ & $\begin{array}{l}34.01 \% \\
(219 / 644)\end{array}$ & & $\begin{array}{l}1.40(0.89 \\
2.20)\end{array}$ & $\begin{array}{l}p= \\
0.146\end{array}$ & $\begin{array}{l}1.34(0.83 \\
2.15)\end{array}$ & $\begin{array}{l}p= \\
0.227\end{array}$ \\
\hline & & $\begin{array}{l}\text { Tertile } \\
3\end{array}$ & $\begin{array}{l}36.99 \% \\
(54 / 146)\end{array}$ & $\begin{array}{l}28.89 \% \\
(186 / 644)\end{array}$ & & $\begin{array}{l}1.75(1.12, \\
2.75)\end{array}$ & $\begin{array}{l}p= \\
0.015^{*}\end{array}$ & $\begin{array}{l}1.43(0.88 \\
2.34)\end{array}$ & $\begin{array}{l}p= \\
0.153\end{array}$ \\
\hline & \multirow[t]{3}{*}{ WHR } & $\begin{array}{l}\text { Tertile } \\
1\end{array}$ & $\begin{array}{l}19.17 \% \\
(28 / 146)\end{array}$ & $\begin{array}{l}36.34 \% \\
(234 / 644)\end{array}$ & $\begin{array}{l}p< \\
0.001 *\end{array}$ & reference & & reference & \\
\hline & & $\begin{array}{l}\text { Tertile } \\
2\end{array}$ & $\begin{array}{l}32.88 \% \\
(48 / 146)\end{array}$ & $\begin{array}{l}32.61 \% \\
(210 / 644)\end{array}$ & & $\begin{array}{l}1.92(1.16, \\
3.17)\end{array}$ & $\begin{array}{l}p= \\
0.011 *\end{array}$ & $\begin{array}{l}1.89(1.13, \\
3.16)\end{array}$ & $\begin{array}{l}p= \\
0.016 \text { * }\end{array}$ \\
\hline & & $\begin{array}{l}\text { Tertile } \\
3\end{array}$ & $\begin{array}{l}47.95 \% \\
(70 / 146)\end{array}$ & $\begin{array}{l}31.05 \% \\
(200 / 644)\end{array}$ & & $\begin{array}{l}3.03(1.88, \\
4.91)\end{array}$ & $\begin{array}{l}\mathrm{p}< \\
0.001 *\end{array}$ & $\begin{array}{l}2.62(1.58, \\
4.33)\end{array}$ & $\begin{array}{l}p< \\
0.001 *\end{array}$ \\
\hline \multicolumn{10}{|c|}{ Data were mean \pm SD or median (IQR) for skewed variables or numbers (proportions) for categorical variables } \\
\hline \multicolumn{10}{|c|}{ * indicate significance of $P$ value $(P<0.05)$} \\
\hline
\end{tabular}


Results are similar in females. In the age-adjusted model, compared to subjects in the lowest WHtR tertile, the risk of prediabetes was 1.90 times higher for subjects in the second tertile $(95 \% \mathrm{Cl} 1.34-2.71, \mathrm{p}<0.001)$ and 2.34 times higher for subjects in the highest tertile $(95 \% \mathrm{Cl} 1.65-3.33, \mathrm{p}<0.001)$. After controlling potential confounders, the relationship between anthropometric indices and prediabetes decreased but remained significant, except for WHR. In the multivariate-adjusted model, the OR of WHtR was the highest. The results are different in the group of males. In the age-adjusted model, all four anthropometric indices were significantly different between the prediabetic and normal blood glucose groups. In the multivariate-adjusted model, only WHR has obvious difference. Compared to subjects in the lowest WHR tertile, the risk of prediabetes was 1.89 times higher for subjects in the second tertile $(95 \% \mathrm{Cl} 1.13-$ $3.16, p=0.016)$ and 2.62 times higher for subjects in the highest tertile $(95 \% \mathrm{Cl} 1.58-4.33, \mathrm{p}<0.001)$.

\section{Receiver operating characteristic (ROC) curve analysis}

ROC curve analyses was performed to compare the predictive values of the anthropometric measures. The respective areas under the ROC curves (AUC) was calculated for the prediction of prediabetes. The results are presented in Table 5 and Fig. 2. In the general population, the AUC of WHtR, BMI, WC and WHR were $0.598,0.593,0.589$ and 0.588 ,

respectively, and the WHtR was the strongest predictor of prediabetes $(p<0.001)$. In females, the AUC of WHtR was the largest $(A U C=0.600)$ compared with other anthropometric values, including BMI $(A U C=0.592), W C(A U C=0.593)$ and WHR (AUC = 0.577). It was also larger than it was in the general population. Although the AUC of WHtR was the largest both in the general population and in the group of females, this was not true in the group of males. The results showed that WHR (AUC $=0.626)$ had a higher predictive value than other indices in males. The AUC of WHtR, BMI and WC were $0.590,0.592$ and 0.575 , respectively.

Table 5

AUC and corresponding 95\% $\mathrm{Cl}$ of different anthropometric indices in men and women

\begin{tabular}{|c|c|c|c|c|c|c|}
\hline \multirow[t]{2}{*}{ Variables } & \multicolumn{2}{|l|}{ Total } & \multicolumn{2}{|l|}{ Female } & \multicolumn{2}{|l|}{ Male } \\
\hline & $\operatorname{AUC}(95 \% \mathrm{Cl})$ & p-value & $\operatorname{AUC}(95 \% \mathrm{Cl})$ & p-value & $\operatorname{AUC}(95 \% \mathrm{Cl})$ & p-value \\
\hline WHtR & $\begin{array}{l}0.598(0.569 \\
0.626)\end{array}$ & $\begin{array}{l}\mathrm{p}< \\
0.001 *\end{array}$ & $\begin{array}{l}0.600(0.564 \\
0.635)\end{array}$ & $\begin{array}{l}\mathrm{p}< \\
0.001 *\end{array}$ & $\begin{array}{l}0.590(0.540 \\
0.640)\end{array}$ & $\begin{array}{l}p= \\
0.001 *\end{array}$ \\
\hline BMI & $\begin{array}{l}0.593(0.564 \\
0.622)\end{array}$ & $\begin{array}{l}\mathrm{p}< \\
0.001 *\end{array}$ & $\begin{array}{l}0.592(0.556 \\
0.627)\end{array}$ & $\begin{array}{l}\mathrm{p}< \\
0.001 *\end{array}$ & $\begin{array}{l}0.592(0.541 \\
0.643)\end{array}$ & $\begin{array}{l}p= \\
0.001 *\end{array}$ \\
\hline WC & $\begin{array}{l}0.589(0.560 \\
0.617)\end{array}$ & $\begin{array}{l}p< \\
0.001 *\end{array}$ & $\begin{array}{l}0.593(0.558 \\
0.628)\end{array}$ & $\begin{array}{l}\mathrm{p}< \\
0.001 *\end{array}$ & $\begin{array}{l}0.575(0.525 \\
0.626)\end{array}$ & $\begin{array}{l}p= \\
0.004 *\end{array}$ \\
\hline WHR & $\begin{array}{l}0.588(0.559 \\
0.618)\end{array}$ & $\begin{array}{l}p< \\
0.001 *\end{array}$ & $\begin{array}{l}0.577(0.541 \\
0.612)\end{array}$ & $\begin{array}{l}p< \\
0.001 *\end{array}$ & $\begin{array}{l}0.626(0.575 \\
0.676)\end{array}$ & $\begin{array}{l}p< \\
0.001 *\end{array}$ \\
\hline
\end{tabular}

\section{Optimal cut-off points of anthropometric indices in the prediction of prediabetes}

Table 6 shows the best cut-off points and corresponding sensitivity and specificity of each index in the prediction of prediabetes. For females, the optimal cut-off point of $\mathrm{WHtR}$ was 0.48 , with the largest Youden index and highest sensitivity, BMI was $23.5 \mathrm{~kg} / \mathrm{m}^{2}$, WC was $75.6 \mathrm{~cm}$, WHR was 0.84 ; for males, the best cut-off point of WHtR was 0.50 , BMI was $25.5 \mathrm{~kg} / \mathrm{m}^{2}$, WC was $88.9 \mathrm{~cm}$, WHR was 0.93 . 
Table 6

Cutoff point for different anthropometric indices in predicting prediabetes

\begin{tabular}{|lllllllll|}
\hline Variables & Female & \multicolumn{7}{c|}{ Male } \\
& $\begin{array}{l}\text { Cutoff } \\
\text { point }\end{array}$ & Sensitivity & Specifity & $\begin{array}{l}\text { Youden } \\
\text { index }\end{array}$ & $\begin{array}{l}\text { Cutoff } \\
\text { point }\end{array}$ & Sensitivity & Specifity & $\begin{array}{l}\text { Youden } \\
\text { index }\end{array}$ \\
\hline WHtR & 0.4795 & 76.2 & 40.5 & 0.167 & 0.4956 & 68.5 & 45.5 & 0.14 \\
\hline BMI(kg/m2) & 23.5273 & 75.1 & 41.1 & 0.162 & 25.4657 & 65.1 & 53.9 & 0.19 \\
\hline WC(cm) & 75.75 & 74.4 & 39.9 & 0.143 & 88.9 & 45.9 & 67.2 & 0.131 \\
\hline WHR & 0.8399 & 70.8 & 43.1 & 0.139 & 0.9259 & 52.7 & 65.5 & 0.182 \\
\hline
\end{tabular}

\section{Discussion}

\section{Main findings}

To the best of our knowledge, this is the first study to compare the function of these four anthropometric indices to predict prediabetes in a long-term cohort study. The present study found that WHtR is the best predictors of the development of prediabetes in a general population and in females in 7 years in advance compared with WC, BMI and WHR, while the WHR can predict the development of prediabetes in males compared with other indices. We determined the optimal cut-off values of these four variables for the prediction of the development of prediabetes in this specific population. For females, the optimal cut-off point of WHtR was 0.48 , BMI was $23.5 \mathrm{~kg} / \mathrm{m}^{2}$, WC was $75.6 \mathrm{~cm}$ and WHR was 0.84 ; for males, the best cut-off point of WHtR was 0.50 , BMI was $25.5 \mathrm{~kg} / \mathrm{m}^{2}$, WC was $88.9 \mathrm{~cm}$ and WHR was 0.93. Early identification of prediabetes can better prevent diabetes.

\section{The ability of WHtR, BMI, WC and WHR to predict prediabetes in general population and female}

Previous studies comparing WHtR, WC, BMI and WHR have been inconsistent in the detection of diabetes ${ }^{[16-18]}$, while few studies analyzed the relationship between these indicators and prediabetes. Other studies have shown that more body fat and higher metabolic risk were found for a given BMI value than European individuals ${ }^{[19]}$, and BMI does not provide details on body fat distribution.

Results in our study were consistent with above opinions. As in other studies, we found that WHtR in females was the best predictor of incident prediabetes. F. Javier Sangro et al ${ }^{[20]}$ studied the association of general and abdominal obesity with prediabetes among 2022 participants in the PREDAPS study, and they found that, compared with BMI among women, the WHtR showed a stronger association with prediabetes, and the results were different between men and women. This can be explained by the following reason, abdominal obesity can induce a state of insulin resistance, which lead to a defective response to insulin in peripheral tissue and result in altered glucose uptake and utilization ${ }^{\text {[20- }}$ 22]. A study from Jordan revealed that the WHtR had a strong association and performed better for the detection of high FBG than the other anthropometric indices ${ }^{[23]}$. An study conducted among Iranian found that among women, compared with the use of BMI, hip circumference and WC, the WHtR was the only significant anthropometric predictor of prediabetes $^{[24]}$. Additionally, the study on the relationship between anthropometric measurements with their best cut-off values and dysglycemia found that WHtR had the highest sensitivity ${ }^{[25]}$. The differences in these results indicated that the validity of anthropometric measurements for the prediction of prediabetes development varies among different genders, ethnicities, and regions. In our study, WHtR was the most effective indices for the prediction of prediabetes in 
females, followed by BMI. WC and WHR were relatively weak predictors. While in males, WHR was the most useful measurement in predicting prediabetes, followed by BMI, WHtR and WC.

Our results in male showed that compared with the function of WHtR, BMI and WC, WHR was the best predictor of incident prediabetes in males. A high WHR is an important risk factor for hyperglycemia. The study of Manju Bala[26] found that WHR is positively correlated with $\mathrm{HbA1c}$, which means that a decrease in WHR is effective for the prevention of prediabetes. The previous findings regarding the association between the WHR and prediabetes ${ }^{[27,28]}$ were consistent with the results of our study.

\section{The best cut-off points for these four anthropometric indices}

It is well known that a single set of cut-offs cannot be applied universally, and there is substantial evidence to show that Asian individuals need lower anthropometric cut-offs for the identification of obesity and abdominal obesity than European individuals ${ }^{[29]}$. Interestingly, in the present study, the best cut-off points of these four anthropometric measurements for the detection of prediabetes differ from those used for the diagnosis of obesity. In addition, it is also smaller than those in other studies, especially in WHtR, BMI and WC, which means that if we do not use a specific value, the frequency of prediabetes may be underestimated. Better control of WHtR, BMI and WC below the diagnosis value of obesity can better prevent the occurrence of prediabetes. While the value of WHR in the present study was bigger than previous. It is maybe because aging can both increase body fat and change the distribution. Therefore, the need to determine specific cut-off points of these variables for optimal screening of prediabetes is urgent. An obvious difference was observed in these four indices in different gender, suggesting that sex-specific values should be recommended for use in practice. This need has also been illustrated in a previous study ${ }^{[30-32]}$. In the present study, the cut-off points of WHtR, BMI and WC were smaller than those that are currently recommended for use in this population, which means that the specific cut-off points can play a role in early prevention.

\section{The ability of WHtR, BMI, WC and WHR to predict diabetes}

Although a recent meta-analysis ${ }^{[33]}$, a prospective study in Iran $^{[34]}$ and some articles ${ }^{[35]}$ indicated that WHtR might be a better predictor of T2D, our study found that there are no significant relationship between any obesity indices and incident diabetes in either sex. The results were the same as those of Bozorgmanesh ${ }^{[36]}$, who studies the relationship between central-obesity indices and incident diabetes. This may be because the follow-up time in most of the literature is not long enough. More long-term follow-up data are needed to further analyze the relationship between traditional anthropometric indices and diabetes.

\section{Limitations}

Some limitations should be addressed in our work. First, the majority of the participants were middle-aged or elderly individuals, so the applicability of this conclusion to the general population remains to be determined. Second, the size of the sample was limited, especially the population of males. More participants will be followed up in the future. Third, there was a lack of OGTT reproducibility. A limitation of financial resources and the unwillingness of subjects were the two major causes of this problem. We tried our best to minimize the associated bias and improve the diagnostic accuracy as much as possible by combining these data with the questionnaire data, FBG and HbA1c results.

\section{Conclusion}

In summary, WHtR is the best predictors of the development of prediabetes in a general population and in females in 7 years in advance, while the WHR can predict the development of prediabetes in males. We determined the optimal 
cut-off values of these four variables for the prediction of the development of prediabetes in this specific population.

Early identification of prediabetes can better prevent diabetes.

\section{Abbreviations}

WHtR

Waist-to-height ratio; BMI:body mass index; WC:waist circumference; WHR:waist-to-hip ratio; FBG:fasting blood glucose; PBG:2 h postload blood glucose; TC:total cholesterol; TG:triglycerides; LDL-C:low-density lipoprotein cholesterol; HDLC:high-density lipoprotein cholesterol; GGT:gamma-glutamyl transferase; HbA1c:hemoglobin A1c; BP:blood pressure; IGT:impaired glucose tolerance; IFG:impaired fasting glucose; SBP:systolic blood pressure; DBP:diastolic blood pressure.

\section{Declarations}

\section{Ethics approval and consent to participate}

The study was approved by the Ethics Committee of Chinese People's Liberation Army General Hospital. Written informed consent was signed by all participants before the investigation. This study adhered to the principles of the Declaration of Helsinki.

\section{Consent for publication}

Not applicable.

\section{Availability of data and materials}

The datasets used to support this study are not freely available in view of participants' privacy protection.

\section{Competing interests}

The authors declare no competing interests.

\section{Funding}

Not applicable.

\section{Authors' contributions}

YM and YG contributed to the conception and design of the study. YG, CZ and PA analyzed the data. YL, KC, LZ, WG, JD, ZL and JB recruited the subjects and supervised the study. YG wrote the initial draft of the paper. YM and YG contributed to the writing, reviewing, and revising of the manuscript.

\section{Acknowledgements:}

Not applicable 


\section{References}

1. Xu Y, Wang L, He J, Bi Y, Li M, Wang T, Wang L, Jiang Y, Dai M, Lu J, Xu M, Li Y, Hu N, Li J, Mi S, Chen CS, Li G, Mu Y, Zhao J, Kong L, Chen J, Lai S, Wang W, Zhao W, Ning G. 2010 China Noncommunicable Disease Surveillance Group. Prevalence and Control of Diabetes in Chinese Adults. JAMA. 2013; 310(9): 948-59.

2. Rydén L, Grant PJ, Anker SD, Berne C, Cosentino F, Danchin N, Deaton C, Escaned J, Hammes HP, Huikuri H, Marre M, Marx N, Mellbin L, Ostergren J, Patrono C, Seferovic P, Uva MS, Taskinen MR, Tendera M, Tuomilehto J, Valensi P, Zamorano JL; ESC Committee for Practice Guidelines (CPG), Zamorano JL, Achenbach S, Baumgartner H, Bax JJ, Bueno H, Dean V, Deaton C, Erol C, Fagard R, Ferrari R, Hasdai D, Hoes AW, Kirchhof P, Knuuti J, Kolh P, Lancellotti P, Linhart A, Nihoyannopoulos P, Piepoli MF, Ponikowski P, Sirnes PA, Tamargo JL, Tendera M, Torbicki A, Wijns W, Windecker S; Document Reviewers, De Backer G, Sirnes PA, Ezquerra EA, Avogaro A, Badimon L, Baranova E, Baumgartner H, Betteridge J, Ceriello A, Fagard R, Funck-Brentano C, Gulba DC, Hasdai D, Hoes AW, Kjekshus JK, Knuuti J, Kolh P, Lev E, Mueller C, Neyses L, Nilsson PM, Perk J, Ponikowski P, Reiner Z, Sattar N, Schächinger V, Scheen A, Schirmer H, Strömberg A, Sudzhaeva S, Tamargo JL, Viigimaa M, Vlachopoulos C, Xuereb RG. ESC Guidelines on diabetes, prediabetes, and cardiovascular diseases developed in collaboration with the EASD. Eur Heart J. 2013; 34(39): 3035-87.

3. Tanne D, Koren-Morag N, Goldbourt U. Fasting plasma glucose and risk of incident ischemic stroke or transient ischemic attacks: a prospective cohort study. Stroke. 2004; 35(10): 2351-5.

4. Norhammar A, Tenerz A, Nilsson G, Hamsten A, Efendíc S, Rydén L, Malmberg K. Glucose metabolism in patients with acute myocardial infarction and no previous diagnosis of diabetes mellitus: a prospective study. Lancet. 2002; 359(9324): 2140-4.

5. Phillips CM, Tierney AC, Perez-Martinez P, Defoort C, Blaak EE, Gjelstad IM, Lopez-Miranda J, Kiec-Klimczak M, Malczewska-Malec M, Drevon CA, Hall W, Lovegrove JA, Karlstrom B, Risérus U, Roche HM. Obesity and body fat classification in the metabolic syndrome: impact on cardiometabolic risk metabotype. Obesity (Silver Spring). 2013; 21(1): E154-61.

6. Flegal KM, Graubard BI, Williamson DF, Gail MH. Excess deaths associated with underweight, overweight, and obesity. JAMA. 2005, 293(15): 1861-7.

7. Flegal KM, Graubard BI, Williamson DF, Gail MH. Cause-specific excess deaths associated with underweight, overweight, and obesity. JAMA. 2007, 298(17): 2028-37.

8. Adams KF, Schatzkin A, Harris TB, Kipnis V, Mouw T, Ballard-Barbash R, Hollenbeck A, Leitzmann MF. Overweight, obesity, and mortality in a large prospective cohort of persons 50 to 71 years old. N Engl J Med. 2006; 355(8): 763-78.

9. Jee SH, Sull JW, Park J, Lee SY, Ohrr H, Guallar E, Samet JM. Body-mass index and mortality in Korean men and women. N Engl J Med. 2006; 355(8): 779-87.

10. Romero-Corral A, Montori VM, Somers VK, Korinek J, Thomas RJ, Allison TG, Mookadam F, Lopez-Jimenez F. Association of body weight with total mortality and with cardiovascular events in coronary artery disease: a systematic review of cohort studies. Lancet. 2006; 368(9536): 666-78.

11. World Health Organization. Obesity: preventing and managing the global epidemic. Report of a WHO consultation, 2000.

12. Hoffman DJ, Wang Z, Gallagher D, Heymsfield SB. Comparison of visceral adipose tissue mass in adult African Americans and Whites. Obes Res. 2005; 13(1): 66-74.

13. Katzmarzyk PT, Bray GA, Greenway FL, Johnson WD, Newton Jr RL, Ravussin E, Ryan DH, Smith SR, Bouchard C. Racial differences in abdominal depotspecific adiposity in white and African American adults. Am J Clin Nutr. 
2010; 91(1): 7-15.

14. Yang W, Lu J, Weng J, Jia W, Ji L, Xiao J, Shan Z, Liu J, Tian H, Ji Q, Zhu D, Ge J, Lin L, Chen L, Guo X, Zhao Z, Li Q, Zhou Z, Shan G, He J. Prevalence of diabetes among men and women in China. N Engl J Med. 2010; 362(12): 1090-101.

15. Razak F, Anand S, Vuksan V, Davis B, Jacobs R, Teo KK, Yusuf S. Ethnic differences in the relationships between obesity and glucose-metabolic abnormalities: a cross-sectional population-based study. Int J Obes (Lond). 2005; 29(6): 656-67.

16. Xin Z, Liu C, Niu WY, Feng JP, Zhao L, Ma YH, Hua L, Yang JK. Identifying obesity indicators which best correlate with type 2 diabetes in a Chinese population. BMC Public Health. 2012; 12: 732.

17. Jayawardana R, Ranasinghe P, Sheriff MH, Matthews DR, Katulanda P. Waist to height ratio: A better anthropometric marker of diabetes and cardio-metabolic risks in South Asian adults. Diabetes Res Clin Pract. 2013; 99(3): 292-9.

18. Harke SM, Khadke SP, Ghadge AA, Manglekar AS, Shah SS, Diwan AG, Kuvalekar AA. Adipocytokines and anthropometric measures in type 2 diabetes. Diabetes Metab Syndr. 2017; 11(suppl 1): S273-S276.

19. Misra A, Khurana L. Obesity-related non-communicable diseases: South Asians vs White Caucasians. Int J Obes. 2011; 35(2): 167-87.

20. Sangrós FJ, Torrecilla J, Giráldez-García C, Carrillo L, Mancera J, Mur T, Franch J, Díez J, Goday A, Serrano R, García-Soidán FJ, Cuatrecasas G, Igual D, Moreno A, Millaruelo JM, Carramiñana F, Ruiz MA, Pérez FC, Iriarte Y, Lorenzo Á, González M, Álvarez B, Barutell L, Mayayo MS, Del Castillo M, Navarro E, Malo F, Cambra A, López R, Gutiérrez MÁ, Gutiérrez L, Boente C, Mediavilla JJ, Prieto L, Mendo L, Mansilla MJ, Ortega FJ, Borras A, Sánchez LG, Obaya JC, Alonso M, García F, Gutiérrez ÁT, Hernández AM, Suárez D, Álvarez JC, Sáenz I, Martínez FJ, Casorrán A, Ripoll J, Salanova A, Marín MT, Gutiérrez F, Innerárity J, Álvarez MDM, Artola S, Bedoya MJ, Poveda S, Álvarez F, Brito MJ, Iglesias R, Paniagua F, Nogales P, Gómez Á, Rubio JF, Durán MC, Sagredo J, Gijón MT, Rollán MÁ, Pérez PP, Gamarra J, Carbonell F, García-Giralda L, Antón JJ, de la Flor M, Martínez R, Pardo JL, Ruiz A, Plana R, Macía R, Villaró M, Babace C, Torres JL, Blanco C, Jurado Á, Martín JL, Navarro J, Sanz G, Colas R, Cordero B, de Castro C, Ibáñez M, Monzón A, Porta N, Gómez MDC, Llanes R, Rodríguez JJ, Granero E, Sánchez M, Martínez J, Ezkurra P, Ávila L, de la Sen C, Rodríguez A, Buil P, Gabriel P, Roura P, Tarragó E, Mundet X, Bosch R, González JC, Bobé MI, Mata M, Ruiz I, López F, Birules M, Armengol O, de Miguel RM, Romera L, Benito B, Piulats N, Bilbeny B, Cabré JJ, Cos X, Pujol R, Seguí M, Losada C, de Santiago AM, Muñoz P, Regidor E. Association of General and Abdominal Obesity With Hypertension, Dyslipidemia and Prediabetes in the PREADPS Study. Rev Esp Cardiol (Engl Ed). 2018; 71(3): 170-7.

21. Hayashi T, Boyko EJ, Leonetti DL, McNeely MJ, Newell-Morris L, Kahn SE, Fujimoto WY. Visceral adiposity and the risk of impaired glucose tolerance: a prospective study among Japanese Americans. Diabetes Care. 2003; 26(3): 650-5.

22. Ascaso JF. Obesidad abdominal, Resistencia a la insulina Y riesgo metabolico y vascular. Med Clin (Barc). 2008; 131(10): 380-1.

23. Morison KM, Xu L, Tranopolosky M, Yusuf Z, Atkinson SA, Yusuf S. Screening for dysglycemia in overweight youth presenting for weight management. Diabetes Care. 2012; 35(4): 711-6.

24. Hadaegh F, Derakhshan A, Zafari N, Khalili D, Mirbolouk M, Saadat N, Azizi F. Pre-diabetes tsunami: incidence rates and risk factors of pre-diabetes and its different phenotypes over 9 years of follow-up. Diabet Med. 2017; 34(1): 6978.

25. Ganguly SS, Sarkar K, Al-Adawi S, Al-Mahrezi A. Screening for dysglycaemia using anthropometric indices in an adult population in Oman. East Mediterr Health J. 2018; 24(3): 254-61.

Page 22/25 
26. Bala M, Meenakshi, Aggarwal S. Correlation of Body Mass Index and Waist/Hip Ratio With Glycated Hemoglobin in Prediabetes. EJFCC. 2019; 30(3): 317-24.

27. Tao LX, Yang K, Huang FF, Liu XT, Li X, Luo YX, Wu LJ, Guo XH. Association of waist circumference gain and incident prediabetes defined by fasting glucose: a seven-year longitudinal study in Beijing, China. Int J Environ Res Public Health. 2017; 14(10): 1208.

28. Worede A, Alemu S, Gelaw YA, Abebe M. The prevalence of impaired fasting glucose and undiagnosed diabetes mellitus and associated risk factors among adults living in a rural Koladiba Town, Northwest Ethiopia. BMC Res Note. $2017 ; 10(1): 251$.

29. WHO/IASO/IOTF. The Asia-Pacific Perspective: Redefining Obesity and its Treatment. 2000.

30. Berber A, Go'mez-Santos R, Fangha“nel G, Sánchez-Reyes L. Anthropometric indices in the prediction of type 2 diabetes mellitus, hypertension and dyslipidaemia in a Mexican population. Int J Obes Relat Metab Disord. 2001; 25(12): 1794-9.

31. Hsu WC, Araneta MR, Kanaya AM, Chiang JL, Fujimoto W. BMI cut points to identify at-risk Asian Americans for type 2 diabetes screening. Diabetes Care. 2015; 38(1): 150-8.

32. Bermúdez V, Salazar J, Rojas J, Calvo M, Rojas M, Chávez-Castillo M, Añez R, Cabrera M. Diabetes and Impaired Fasting Glucose Prediction Using Anthropometric Indices in Adults from Maracaibo City, Venezuela. J Community Health. 2016; 41(6): 1223-33.

33. Wagner DR, Heyward VH. Measures of body composition in blacks and white: a comparative review. Am J Clin Nutr. 2000; 71(6): 1392-402.

34. Bozorgmanesh M, Hadaegh F, Ghaffari S, Harati H, Azizi F. A simple risk score effectively predicted type 2 diabetes in Iranian adult population: population-based cohort study. Eur J Public Health. 2011; 21(5): 544-9.

35. Hou X, Chen S, Hu G, Chen P, Wu J, Ma X, Yang Z, Yang W, Jia W. Stronger associations of waist circumference and waist-to-height ratio with diabetes than BMI in Chinese adults. Diabetes Res Clin Pract. 2019; 147: 9-18.

36. Bozorgmanesh M, Hadaegh F, Zabetian A, Azizi F. Impact of hip circumference and height on incident diabetes: results from 6-year follow-up in the Tehran Lipid and Glucose Study. Diabet Med. 2011; 28(11): 1330-6.

\section{Figures}




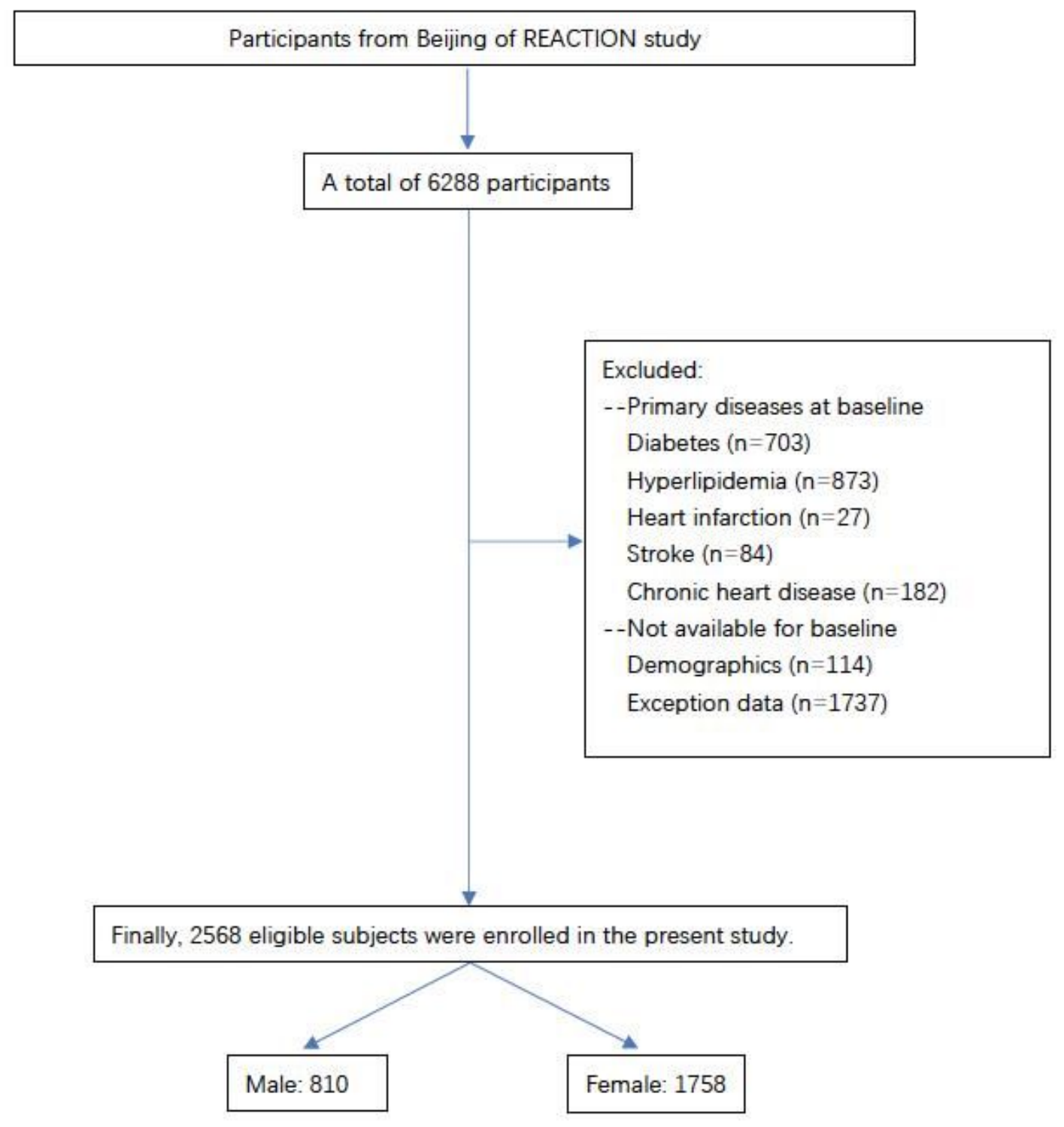

Figure 1

Flow chart of the selection of study participants

A

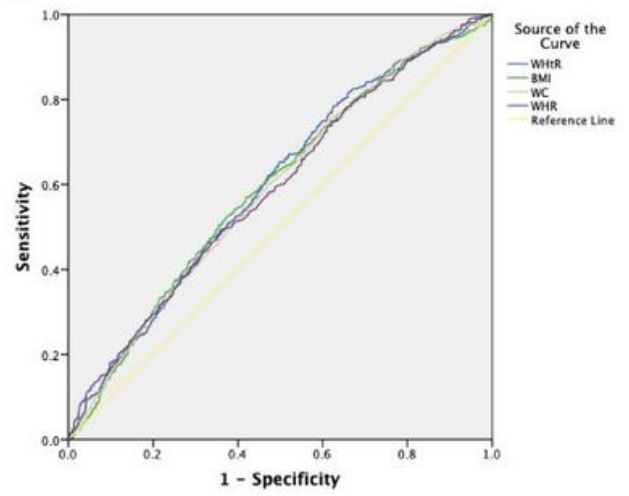

B

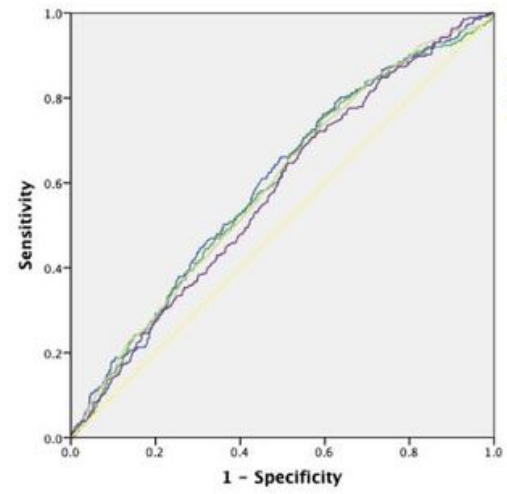

C

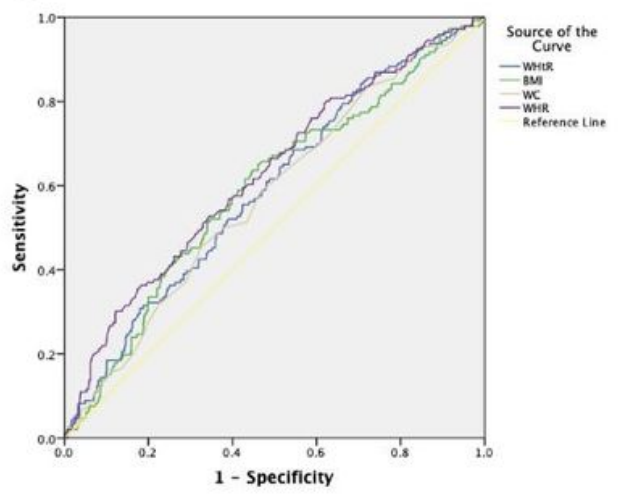

Figure 2 
The ROC curve of these four anthropometric indices in general population, female and male A: The ROC curve in general population. B: The ROC curve in female. C: The ROC curve in male.

\section{Supplementary Files}

This is a list of supplementary files associated with this preprint. Click to download.

- SupplementTable2.docx

- SupplementTable1.docx 